\title{
A Multi-Range Architecture for Collision-Free Off-Road Robot Navigation
}

\author{
Pierre Sermanet $^{1,2}$ Raia Hadsell $^{1}$ Marco Scoffier $^{1,2}$ Matt Grimes $^{1}$ \\ Jan Ben ${ }^{2}$ Ayse Erkan ${ }^{1}$ Chris Crudele ${ }^{2}$ Urs Muller $^{2}$ Yann LeCun $^{1}$ \\ (1) Courant Institute of Mathematical Sciences \\ New York University \\ New York, NY USA \\ (2) Net-Scale Technologies \\ Morganville, NJ USA
}

\begin{abstract}
We present a multi-layered mapping, planning, and command execution system developed and tested on the LAGR mobile robot. Key to robust performance under uncertainty is the combination of a short-range perception system operating at high frame rate and low resolution and a long-range, adaptive vision system operating at lower frame rate and higher resolution. The short-range module performs local planning and obstacle avoidance with fast reaction times, while the long-range module performs strategic visual planning. Probabilistic traversability labels provided by the perception modules are combined and accumulated into a robot-centered hyperbolic-polar map with a 200 meter effective range. Instead of using a dynamical model of the robot for short-range planning, the system uses a large lookup table of physically-possible trajectory segments recorded on the robot in a wide variety of driving conditions. Localization is performed using a combination of GPS, wheel odometry, IMU, and a high-speed, low-complexity rotational visual odometry module. The end to end system was developed and tested on the LAGR mobile robot, and was verified in independent government tests.
\end{abstract}

\section{Introduction}

Walking through a park, a forest, or a mountain trail, humans and animals combine information at many different spatial resolutions and on many different time scales to help them plan a trajectory. The general direction is determined by the heading of the goal, combined with cues from longrange vision, and sometimes constrained by the direction of the trail being followed. Long-range visual cues are refined over time on a relatively slow time scale, by combining information from multiple viewpoints as the subject moves along. The long-range plan is updated periodically, whenever the accumulated evidence calls for it. Short-range planning, on the other hand, requires constant attention and fast reaction times. Nearby obstacles must be detected quickly, so that the internal representation of the immediate surroundings can be updated on a fast time scale. The map of the immediate surroundings must have considerably higher spatial resolution than the map of far away locations, but this high-resolution is somewhat ephemeral: we quickly forget the details of all the obstacles we circumvented. This accords with studies of human subjects who have been shown to focus on nearby objects much more frequently than on distant areas (Wagner et al., 1980). Occasional distant gazing suffices to maintain a global trajectory, but frequent nearby gazing is 
necessary for obstacle avoidance.

This motivates the design of navigation systems for autonomous robots in which a number of perception-mapping-planning-control loops operate at different ranges, different spatial resolutions, different image resolutions, and different time scales. A fast control loop with low latency and high frame rate constructs a short-range map with high-spatial resolution from short-range vision at low image resolution, while a slower control loop with higher latency and lower frame rate constructs a long-range map with low spatial resolution using long-range vision at maximum image resolution. The short-range planning is updated on a fast-time scale and takes the vehicle dynamics into account, while the long-range planning is updated rarely and ignores dynamical constraints.

This paper describes such a multi-timescale, multi-resolution, multi-range architecture for visionbased off-road robot navigation. The system was implemented and tested on the LAGR platform. The present paper concentrates on the architecture and system-level issues, as well as on the fastreacting, short-range vision system, the learning-based dynamical trajectory control, the probabilistic mapping, the multi-range planning strategy, and the localization and odometry subsystems. The adaptive long-range vision is described in full details in a companion paper (Hadsell et al., 2008), and very briefly described in the current paper.

\subsection{Overview}

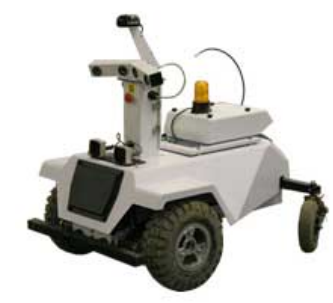

Figure 1: The LAGR platform has no active external sensors, it relies solely on two stereo camera pairs (4 cameras). A GPS, a low-cost IMU, and wheel encoders provide odometry data. It is a differential drive (non-holonomic) off-road vehicle measuring $1.2 \mathrm{~m}$ in length by $0.74 \mathrm{~m}$ in width by $1.02 \mathrm{~m}$ in height. It weighs $109 \mathrm{~kg}$ and can travel at up to $1.3 \mathrm{~meters} / \mathrm{seconds}$. The robot contains three computers with dual-core CPUs: two "eye" machines for vision processing of each stereo pair, and one "planner" machine for planning. A fourth computer takes care of low-level control tasks.

The LAGR robot platform, shown in figure 1, is somewhat unique in the world of outdoors mobile robots in that it uses no active sensors, and relies solely on cameras to sense its environment. This was a deliberate choice in the program designed to promote new advances in robot vision, as well as in the application of machine learning to mobile robotics. The LAGR robot has two forwardlooking stereo camera pairs with a combined field of view of 120 degrees, a GPS, a low-cost Inertial Measurement Unit (IMU), wheel encoders, and a front bumper with bump sensors. It can travel at 1.3 meters/second (fast walking speed).

The implementation of a fully-functional, reliable, and efficient vision-based navigation system for off-road robots such as the LAGR platform is a very difficult challenge for robotics research. In contrast with indoors environment and constrained outdoors environments (roads and trails), off-road scenes and natural obstacles can vary widely in shapes, colors, textures and lighting.

A key question is how to construct a long-range vision system that can reliably label far away objects and regions as traversable or non-traversable, and adapt to new environment. This question is addressed in the companion paper (Hadsell et al., 2008). With the limited resolution, and small 
stereo baseline of the camera pairs, the practical range of stereo-based algorithms is limited to about 15-20 meters. With such short-sightedness, a pure stereo-based system can get trapped in cul-de-sacs, and waste a considerable amount of time trying to find its way around wide obstacles. Furthermore, stereo often produces no depth values (or erroneous ones) for a large proportion of the pixels, when the image is too uniform, too repetitive (like tall grass), or too saturated by the sun or by dark shadows. A long-range monocular vision module is required. Our module is based on a convolutional network that is trained off-line to extract features over large sliding windows in the images, followed by a linear classifier fed with these features and adapted on-line using labels provided by a stereo vision system.

The main topic addressed in the present paper is how to integrate the various long-range and shortrange perception, mapping, planning, and control modules into a coherent architecture.

The system uses three visual perception modules. A first stereo-based system operating at 160x 120 resolution and 5 to 10 frames per second establishes a traversability map within a 5 meter radius from a stereo point cloud. A second stereo system with a 12 meter range uses multiple heuristics to establish medium-range traversability map. Finally, a monocular convolutional network operating at $512 \times 384$ resolution and 1 frame per second estimates the traversability of every overlapping window in the input image at multiple scales. The last layer of the convolutional network is adapted on-line using labels produced by the medium-range stereo system, in accordance with the "nearto-far learning" methodology (Stavens and Thrun, 2006).

A particularly important question is how to cope with the uncertainty in the output of the perception modules, and how to reduce this uncertainty by accumulating evidence about the traversability from multiple perception modules over long periods of time. The perception modules produce two kinds of uncertainties: uncertainty about the category (or traversability label) of a particular region, and uncertainty about the location of that region in relation to the robot.

Distance estimates of far-away objects (e.g. trees) that appear near the horizon in the image are very imprecise. As the robot moves, these distance estimates fluctuate. When using a Cartesian traversability map with fixed-resolution, these objects are often smeared out over many map cells. Our solution is to use a robot-centered hyperbolic-polar map (h-polar map), in which the radial size of a cell increases hyperbolically (to infinity) with the distance from the robot. Such a map can be seen as a virtual image-plane map, since the radial depth of each map cell corresponds to the growing uncertainty of object distances which diverges to infinity as they get closer to the horizon in the image plane.

For each frame, and each pixel in the frame, the perception modules produce a vector of confidence values, with one value for each possible category of the region around the pixel. The categories are super traversable (very smooth terrain), traversable, obstacle foot (the location where an obstacle meets the ground), obstacle, and super obstacle. The confidence vectors produced from a single frame may contain occasional mistakes. Our system accumulates these confidence vectors in the h-polar map as the robot moves and sees the same locations from multiple viewpoints, thereby reducing the uncertainty and the noise of individual classifications. Before planning, the map confidence histograms are transformed into traversability costs.

A important remaining question is how to integrate the dynamical constraints of the robot into a fast-reacting, short-range planning. Our system uses a large dictionary (or lookup table) of initial trajectory snippets (also known as maneuvers) with a $2.5 \mathrm{~m}$ radius that have been collected by driving the robot under human control in a wide variety of conditions. 


\subsection{Related Work}

Learning algorithms allow robots to adapt to new situations and gain experience. Learning can be applied in a number of ways to improve navigation, e.g. learning terrain slippage (Angelova et al., 2006) and traversability (Angelova et al., 2007) to improve mobility or to avoid getting stuck, with human supervision and Bayesian estimates (Ollis et al., 2007), learning depth from monocular images (Saxena et al., 2007) or learning natural and man-made pathways from color and texture (Blas et al., 2008). Learning algorithms can also be used to extend stereo vision range with near-to-far learning, learning the long-range appearance of terrains and obstacles using color features (Albus et al., 2006) with Markov random fields (Vernaza et al., 2008), with suitable features and a linear support vector machine (Bajracharya et al., 2008), or using reverse optical flow (Lookingbill et al., 2007). Typically trained off-line, features can also be learned online to provide a continuous adaptability (Grudic et al., 2007).

A navigation software developed by CMU/NREC, called "Baseline" was used by the government testing team to measure improvements during monthly tests. The Baseline system can be seen as a benchmark representing the state of the art around the end of 2004. It is based on medium-range stereo vision, a Cartesian/grid map containing traversability scores, and an incremental long-range planning algorithm called $\mathrm{D}^{*}$ (Stentz, 1994). In the PerceptOR program developed at CMU, which preceded the LAGR program, a number of the issues that plagued the Baseline system were addressed (Kelly et al., 2006). A multi-range architecture was proposed, but it was only used for planning. A similar maneuver-based local planner was used. Finally, only two levels of planning were used, local and global grid-based planning, while we advocate for an additional intermediate level of long-range but local vision and planning coupled with a global topological planning. A key shortcoming of Cartesian traversability map approaches is that they are very sensitive to the accuracy with which the robot is localized in the map. This is rather unfortunate, since GPS fixes can be quite inaccurate, and even non-existent (e.g. in heavily wooded areas).

\section{System Architecture}

The overall system architecture is depicted in Fig. 2. Each level operates at lower frequency, and higher level than the level below. The left half of the figure contains the perception modules, while the right part contains the planning and control modules.

Layer 1 contains a short-sighted $(5 \mathrm{~m})$ but robust and fast perception module (1-a, called Fast-OD for Fast Obstacle Detection). The module populates a robot-centered Cartesian map (1-b) with a 5 meters radius and a $20 \mathrm{~cm}$ resolution. The module runs a simple stereo algorithm on 160x 120 image pairs. The ground plane is first detected, and a region is marked as obstacle if many points within that region are dangerously above the ground plane. The map is used by the maneuver-based trajectory planning subsystem. The best path from each point on a robot-centered circle with $2.5 \mathrm{~m}$ radius to each point on the $5 \mathrm{~m}$ radius is computed using Dijkstra. Then for each point on the $5 \mathrm{~m}$ radius, the Layer- 2 planning module computes a cost to the goal. The short-range planner searches for maneuvers in the repertoire (logged wheel velocities collected during tele-operation and autonomous navigation) whose initial states match the current dynamical state (wheel speeds) of the robot (1-d). It then picks the maneuver that has the lowest overall cost to the goal while staying clear of lethal obstacles. Accurately estimating the orientation of the robot within the map is paramount. An error in the orientation has catastrophic effects on the crispness of the map, as obstacles get smeared across the map as the robot rotates. Since the wheels often slip on the ground, and the IMU drifts quickly, a simple and efficient rotational visual odometry module (VO) estimates the angle by which the robot has rotated since the last frame (1-c). The VO module tracks 


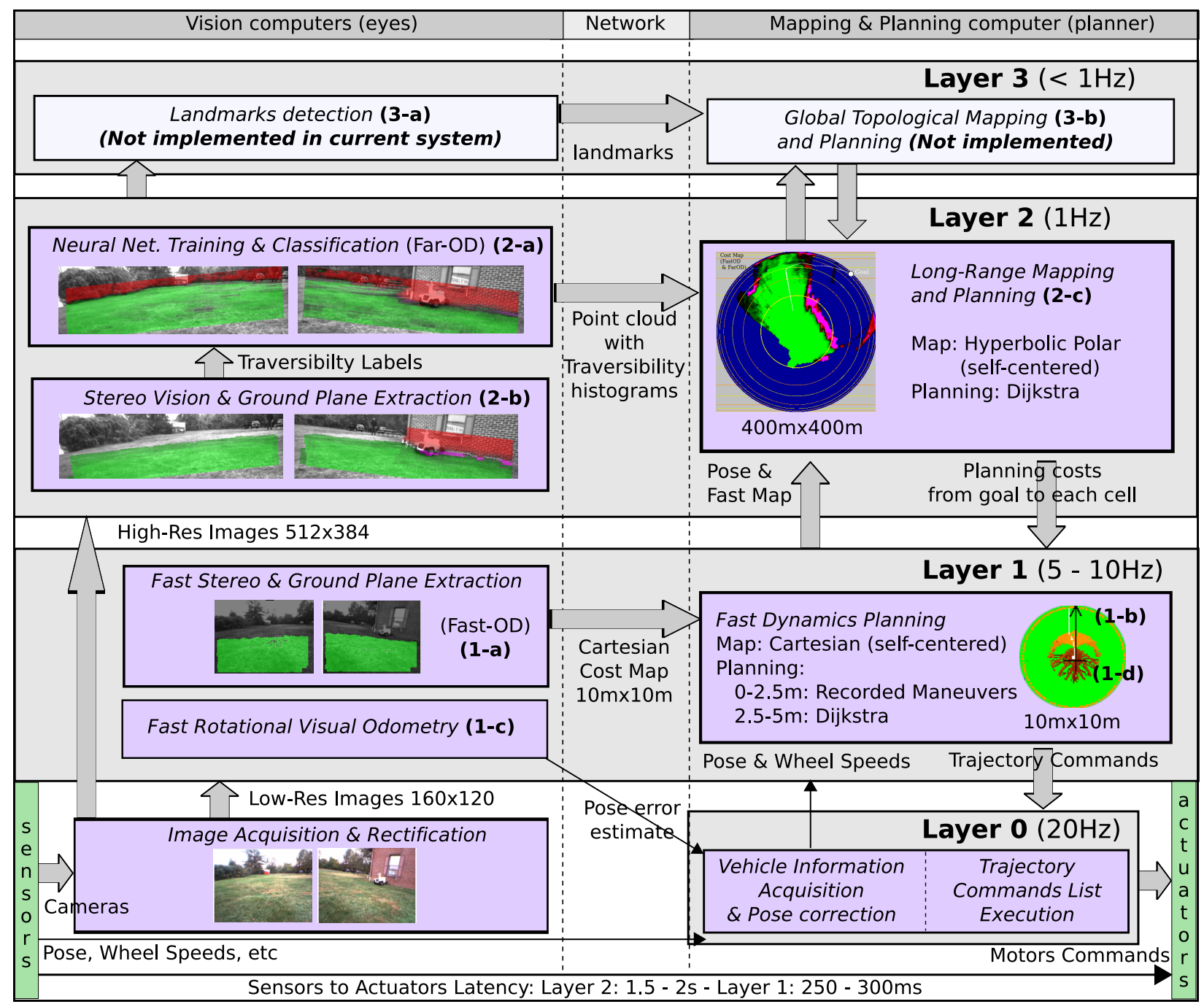

Figure 2: Overall system architecture. Each layer contains perception, mapping and planning modules (except for layer 0). From bottom to top, sensors-actuators latency and period increase while resolutions decrease. Layers 1 and 2 are self-centered while layer 3 is global. Layer 0, also known as control loop, guarantees the flow of motor commands required at $20 \mathrm{~Hz}$. Layer 3 was not implemented in the current system because the scale of the LAGR tests did not require global mapping when our local mapping can handle a $200 \mathrm{~m}$ radius.

a number of distinctive windows on far-away objects near the horizon from one frame to the next. Layer 1 operates at 5 to 10 frames per second.

Layer 2 contains the long-range obstacle detection system (called Far-OD), which is built around a large convolutional network (Hadsell et al., 2008) operating on horizon-leveled image bands extracted from input images at $512 \times 384$ resolution (2-a). The advantages of convolutional networks for this application is that they automatically learn appropriate features representations through supervised or unsupervised training methods, and they are relatively fast and simple. More importantly, they can compute a label for each pixel based on a large input window surrounding the pixel. Therefore, they can go beyond the popular but limited approach to near-to-far learning that uses simple color histogram as inputs. The last layer of the convolutional network, which can be seen as a logistic regression classifier, probabilistically classifies the image regions into five traversability labels. This last layer automatically adapts to new environments as the robot runs. The desired labels are provided to the classifier by a stereo-based obstacle detection system (2-b) with a range of 
12 meters. This system uses multiple ground plane fitting and a number of point-cloud heuristics to produce reliable labels. The vectors of confidences over labels are mapped from their position in the image plane to their corresponding location in the world (or in the h-polar map) using a ground-plane assumption. The confidence vectors are accumulated into histograms stored at their corresponding cell in the h-polar map (2-c). After each frame, the accumulated histograms are mapped to traversability costs, and the Dijkstra planning algorithm is launched. While the radius of the h-polar map and the long-range vision system is infinite in principle, its effective radius is roughly 100 to 200 meters. Overall, Layer 2 operates at one frame per second.

Layer 3 is included in the figure for completeness, but is not implemented in the system described in this paper. Its presence would be necessary for the robot to navigate long courses whose extent goes beyond the effective radius of the h-polar map. It would contain a very long-range topological map (3-b) in which visually distinctive landmarks are detected (3-a) and saved.

By analogy with the navigation mechanism in humans, we know which streets to take to get to a restaurant (Layer 3). We can see the corner to turn two blocks away, and we know which way to turn (Layer 2). We walk there while avoiding bumping into other pedestrians (Layer 1). We occasional think about the global-level route planning (Layer 3), we don't need to look often to the far away end of the block (slow Layer 2) but we will look very often to avoid other pedestrians (fast Layer 1).

\section{Multi-Range Perception and Planning}

Multi-layer architectures (Fig. 2), first introduced by (Brooks, 1986), provide a crucial latency and frequency independence between different levels of planning but also different levels of perception. Key components of each layer are further described. We will first discuss motivation and implementation details for the multi-layer, multi-range architecture.

\subsection{Motivation and Initial Experiments}

As the sophistication and computation load of our advanced vision module was increasing, our initial sequential navigation architecture would cause the robot to collide with an increasing number of obstacles. In order to quantify the relationship between control loop period and system responsiveness in a traditional sequential architecture, we set up a field experiment that would compare the responsiveness of our sequential system as a function of different control loop periods and measure the number of collisions on a fixed course. To ensure that the performance of the system was only affected by the control loop period, we use a single, fixed, mid-range obstacle avoidance module. For each test, the control loop period was artificially increased, but the obstacle avoidance processing and the test course were kept fixed.

The LAGR vehicle was tested in a picnic area at Holmdel Park, NJ. The course had 8 large trees, 2 barrels, and 2 picnic tables, and the goal was 30m away. The start position faced 90 degrees away from the goal and there was a barrel $3 \mathrm{~m}$ from the start. When a run was started, the barrel would not be seen until the robot turned toward the goal, at which point the robot would have to quickly turn to avoid the barrel. The vehicle was tested using 4 different control loop periods (time from frame time-stamp to issuing drive commands): $360 \mathrm{~ms}, 510 \mathrm{~ms}, 660 \mathrm{~ms}$, and $960 \mathrm{~ms}$.

Results of the experiment are summarized in Fig. 3. The shortest loop period performed significantly better than any of the other test modes, particularly in avoiding the first obstacle. The 
intermediate loop periods produced poorer performance: they usually crashed into the first barrel, but could often navigate around other obstacles (although not as consistently as the shortest loop). The longest period $(960 \mathrm{~ms})$ produced abysmal driving, repeatedly crashing into obstacles.

In addition, the latency plots in Fig. 22 show that an incompressible sensor latency of 190ms already handicaps the robot's reactivity. Similarly, inertia of the vehicle as shown by plots of Fig. 15 also add latency to the reactivity. The reactivity latency caused by vehicle dynamics is addressed by maneuver-based planning in the further sections. Although the sensors and processing latencies can also be addressed by estimating delays before and after planning, the vehicle still gains in reactivity by minimizing latencies.

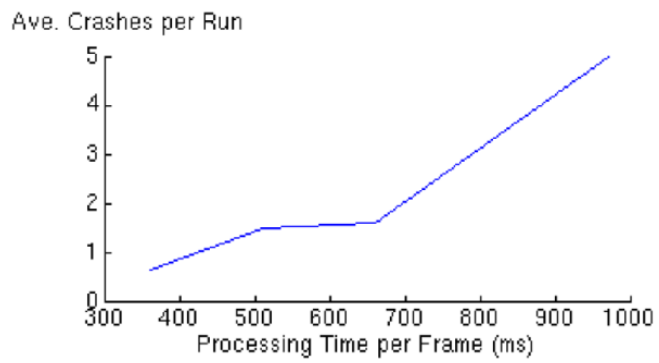

Figure 3: Experimental results: performance of the early sequential system, as measured by the number of collisions per run, for different control loop periods. The performance is roughly linearly correlated with the length of the control loop.

Layered architectures have been used for a long time and this experiment confirms once more the importance of reactivity versus vision range in close combat navigation. (Wagner et al., 1980) investigated the gaze behavior of walking humans in an outdoor environment. The results indicated that the human gaze was directed at objects close to the observer a majority of the time. (Bayouth et al., 1998) proposed a hybrid human-computer layered architecture for highway driving. (Beetz, 2001) described a method for integrating perception, planning, and control in a uniform framework and demonstrates the framework with a service robot. (Low et al., 2002) presented a method for unified planning and motion control for a mobile robot.

\subsection{Implementation Details}

Here we specify what characteristics each module should have for different layers. For example, the naming of the obstacle detection modules (OD) indicate their characteristics: Fast-OD must be fast but does not have to see far away, whereas Far-OD has to see far but not necessarily fast.

\subsubsection{Perception Modules Characteristics}

The perception processes are run on the "eye" machines and transmit the traversability data over the network to the "planner" machine. Because it has to be fast and does not need to be longrange, the resolution of the Fast-OD can be as low as 160x120 compared to traditional systems. Along with the low resolution, stereo processing heuristics producing the traversability map are kept simple in order to guarantee the low latency of the Fast-OD. On the contrary, the Far-OD uses higher resolution input $(512 \times 384)$ to reach as far as possible $(>100 \mathrm{~m})$ but is allowed to be slow. 


\subsubsection{Mapping and Planning Modules Characteristics}

Each planning layer must be able to operate independently of the layer above for some time and produce data for the lower level. For example, in the Layer-2 (hyperbolic polar) map, all best paths from the goal to each cell are computed once per Layer- 2 iteration $(1 \mathrm{~Hz})$ and passed to the Layer-1 planner $(5-10 \mathrm{~Hz})$. At Layer 1 , knowing the current translation and rotation relative to the Layer- 2 map, the globally optimal path can be computed using the locally optimal paths reaching all points at a fixed radius $(5 \mathrm{~m})$. The transposed local candidates cost into the Layer- 2 map are added to the pre-computed paths cost and the minimum sum determines the globally optimal path. When the best local path or trajectory is found, the sequence of motor commands to execute is sent to the Layer-0 control loop $(20 \mathrm{~Hz})$. The control-loop can now operate independently by just iterating through the commands sequence. Since vehicle-centered maps are used, maps must be re-centered at every iteration of the layer before a data update. Thanks to the layered architecture, not only the planning is executed once per level, but also the mapping and re-centering, which is a costly process in case of the hyperbolic polar map. As pointed out earlier, latency from acquisition to planning is minimized as much as possible but is also addressed by re-centering maps on the last known pose. Delays after planning are thus not taken into account and must remain nearly null. Another advantage of using layers is that different planning and mapping schemes best suited for different ranges can easily be combined. For example, the hyperbolic polar mapping is suited for far-range data while Cartesian mapping is more adequate for short-range because it is simple and fast. Similarly, Dijkstra planning is convenient for long-range while dynamics planning is required in the short range. More details about the actual mapping and planning algorithms are given further in the components sections.

\subsubsection{Process Priorities}

When running different layers on a same CPU, the lowest levels require an increasing execution priority as the latency minimization and frequency maximization requirements increase. This can be achieved by using the priority scheduling features of a real-time operating system or with a regular operating system by giving control of the higher level processes to the lower level processes. For example when receiving a new frame to process, the Fast-OD pauses the Far-OD process and resumes it when processing is done. Then the Fast-OD can pause itself for a fixed amount of time to allow some processing cycles to the Far-OD. A trade-off must be found depending on the available CPU budget, running speed and vision distance. At the risk of losing reactivity to moving obstacles, one could extend this idea by giving more CPU cycles to Far-OD when going straight and more to Fast-OD when the robot turns. This custom priority scheduling was successfully tested on the first generation of the LAGR vehicle, but was no longer needed after a hardware upgrade to dual-core CPUs.

The timing plots of Fig 22 show the actual latencies and frequencies obtained for layers 1 and 2 on the dual-core machines.

\section{Layer 2: Long-Range Perception, Mapping and Planning}

We describe in this section the complete Layer 2 of Fig. 2, and in particular the mapping and planning approaches used to address the issues inherent to long-range vision: classification and distance uncertainties and computational efficiency. The mapping method allows the accumulation of evidence from multiple frames in a principled manner. The geometry of the map accurately reflects the range uncertainty associated with image-plane obstacle labeling. It can furthermore represent an effectively infinite radius with a finite number of cells. Lastly, the information in the map allows 

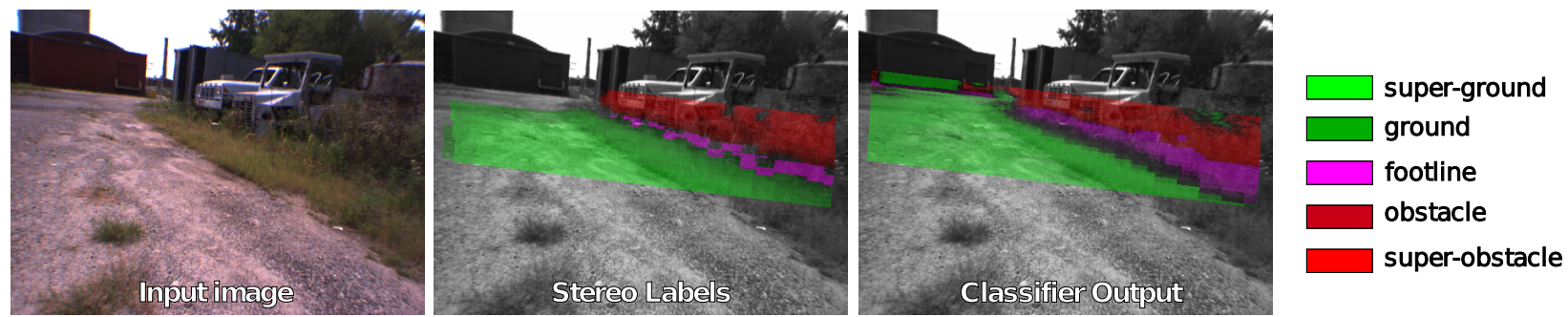

Figure 4: Neural network output: 5 classes. The input image (left) is fed into the neural network for classification at resolution $512 \times 384$, producing traversability labels (overlaid on the right image) up to 100 meters. The network is trained both off-line and online using the stereo labels (center) up to 12 meters for self-supervision.

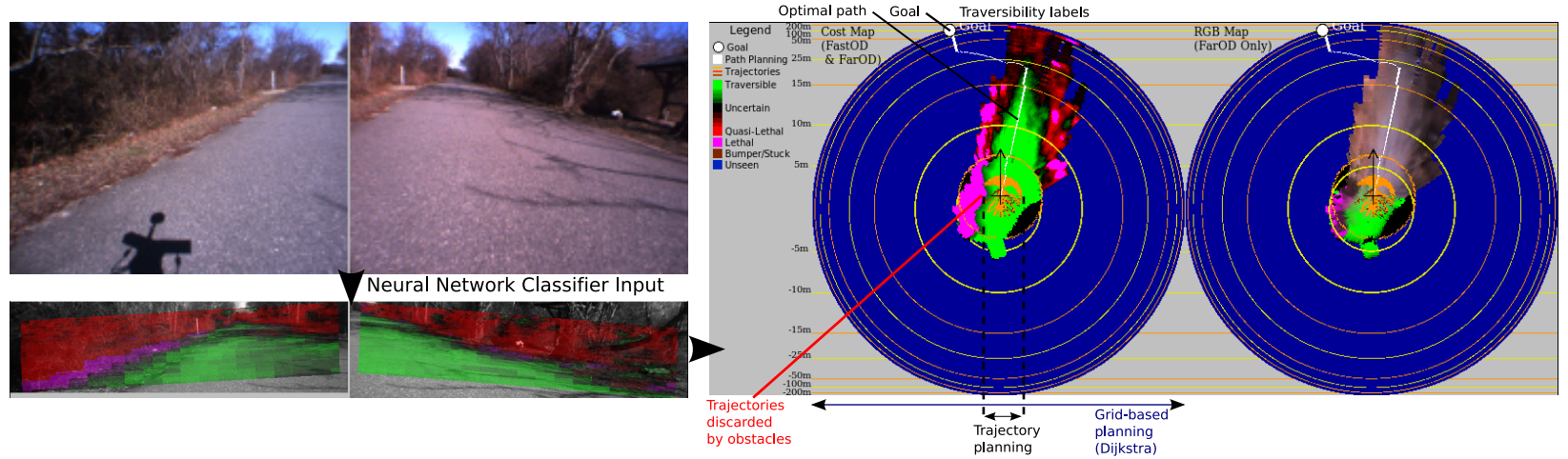

Figure 5: Multi-layer planning with trajectories. Long-range (bottom left) and short-range labels are accumulated in the hpolar map (right). The optimal path in white is computed through multiple planning layers, with Dijsktra at Layer 2 and trajectories (orange curves) planning at Layer 1 on a 10x10m area.

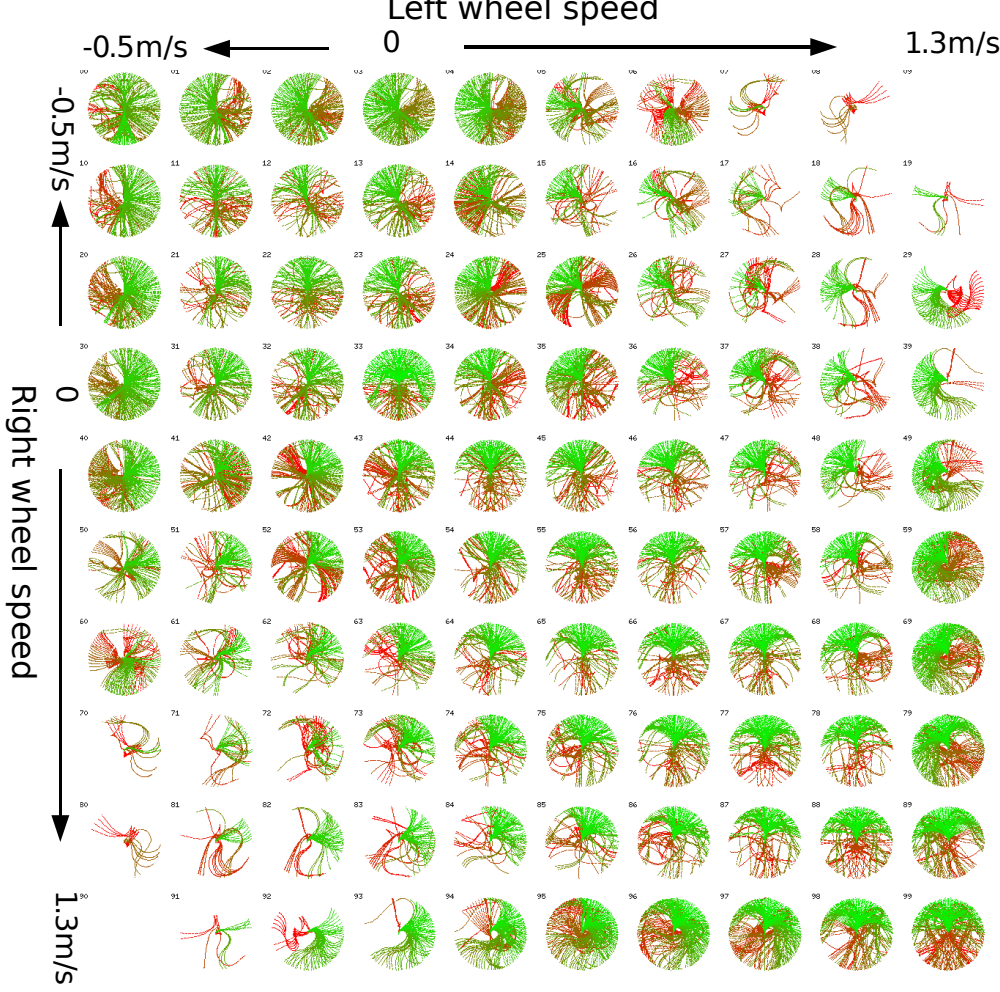

49
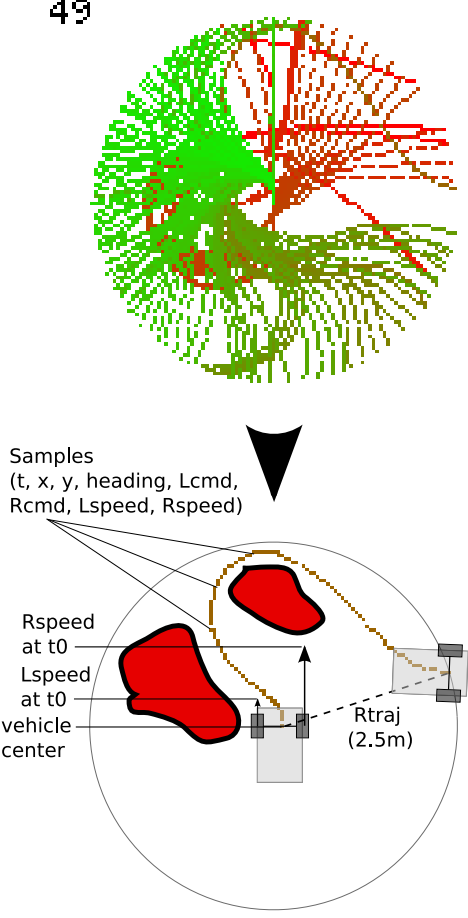

Figure 6: Maneuver dictionary (left) and execution example (right). The maneuver bank shows 100 sets of possible trajectories for each initial speeds. The robot initially goes full speed backwards at the top left corner and full speed forward at the bottom right. For each set, trajectories are colored from green for the fastest ones to reach the $5 \mathrm{~m}$ radius to red for the slower ones, e.g. it is obvious based on colors that turning left when already turning hard left is faster. For example with initial state $(4,9)$ on the right, i.e. initially turning hard left, the robot can only avoid the front obstacle with a large left turn around it. 
to dynamically adjust the planning policy so as to be more aggressive or more conservative. A key element for long-range mapping is the ability to accurately detect the bottom (or foot) of obstacles, allowing to "block" obstacles beyond their foot. By only using the bottom of obstacles, we avoid projecting down on the ground plane obstacles for which no elevation information is available and creating false obstacles.

\subsection{Long-Range Vision}

Our long-range vision learning approach briefly introduced here is fully described in (Hadsell et al., 2008). The existing paradigm for vision-based mobile robots relies on hand-tuned heuristics: a stereo algorithm produces a $(x, y, z)$ point cloud and traversability costs are assigned to points based on their proximity to a ground plane (Kelly and Stentz, 1998; Goldberg et al., 2002). However, stereo algorithms that run in real-time often produce cost-maps that are short-range, sparse, and noisy. Our learning strategy uses these stereo labels for supervision to train a real-time classifier. The classifier then predicts the traversability of all visible areas, from close-range to the horizon. For accurate recognition of ground and obstacle categories, it is best to train on large, discriminative windows from the image, since larger windows give contextual information that is lacking in color and texture features. Other research has explored the use of online learning for mobile robots, but their methods have been largely restricted to simple color/texture correspondences (Manduchi et al., 2003; Sofman et al., 2006).

\subsection{Mapping with Categorical Uncertainty: Histograms}

\subsubsection{Classifier Output}

The network produces a floating-point value for each of the 5 following classes (Fig. 4) detected by the stereo module: super-ground, ground, foot-line, obstacle, super-obstacle. Having more visually-consistent classes helps the network to produce better classification in comparison to a binary classifier as used in previous versions of the system. In addition, it conveniently suits the histogram scheme used in our planning cost decision algorithm described in the following section.

\subsubsection{Label uncertainty: Cost from Histogram}

Label uncertainty can be caused by differences of view points, sensor noise or learning phase of the online neural network. Mapping with label uncertainty in mobile robotics has been addressed by several approaches from Bayesian techniques (Elfes, 1991) to fuzzy logic (Oriolo et al., 1997). Trivial approaches such as using latest labels only or running averages are simple and fast but lack confidence and accuracy and would cause an early fusion of the multi-class network outputs. We use an histogram approach (Fig. 7) suited for multiple classes and able to delay the traversability decision to planning time: each cell contains $K$ bins, each bin corresponding to a class (or a range of traversability for single class outputs). Each new label is merged in a cell through a simple addition. Before planning, the histogram is translated into a traversability cost (Fig. 8). At that time, the traversability decision can be modulated by the current planning policy: conservative vs. aggressive. As label uncertainty increases with distance, a distance decay must be applied to incoming frames. 


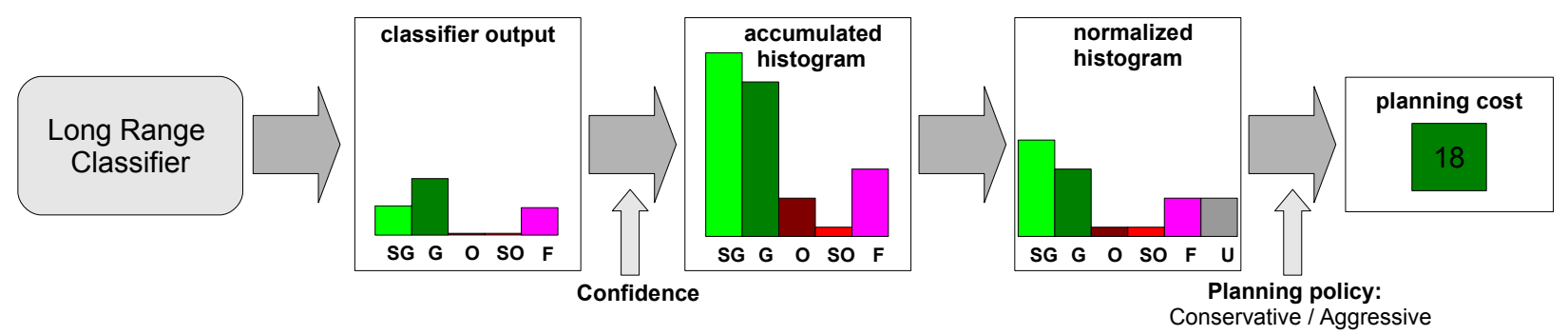

Figure 7: Histogram to cost process. The output of the classifier is multiplied by the current learning confidence and added to an existing histogram. Before converting the histogram to a planning cost, it is normalized. Bin U in the normalized histogram corresponds to constant $c_{\text {uncertain }}$ which brings confidence to 0 when the sum $S$ is small (uncertainty is showed in Fig. 8). Finally, the current planning policy modulates the mapping from the normalized histogram to a single planning cost.

\subsubsection{Histogram to cost transformation}

Let $\sum_{k} \operatorname{bin}_{k}+c_{\text {uncertain }}$ be the sum of all bins of an histogram with an added constant $c_{\text {uncertain }}$ which brings confidence to 0 when the sum is small. Let $\sum_{k} w_{k} b i n_{k}$ be the weighted sum of all bins. $w_{k}$ are weights empirically tuned with real examples. The weight values $w_{k}$ used from superground to foot in the order of Fig 7 are: $-1.0,-0.7,0.7,0.9$ and 1.8. The normalized sum $S$ tuned by gain parameters $\gamma$ is defined by:
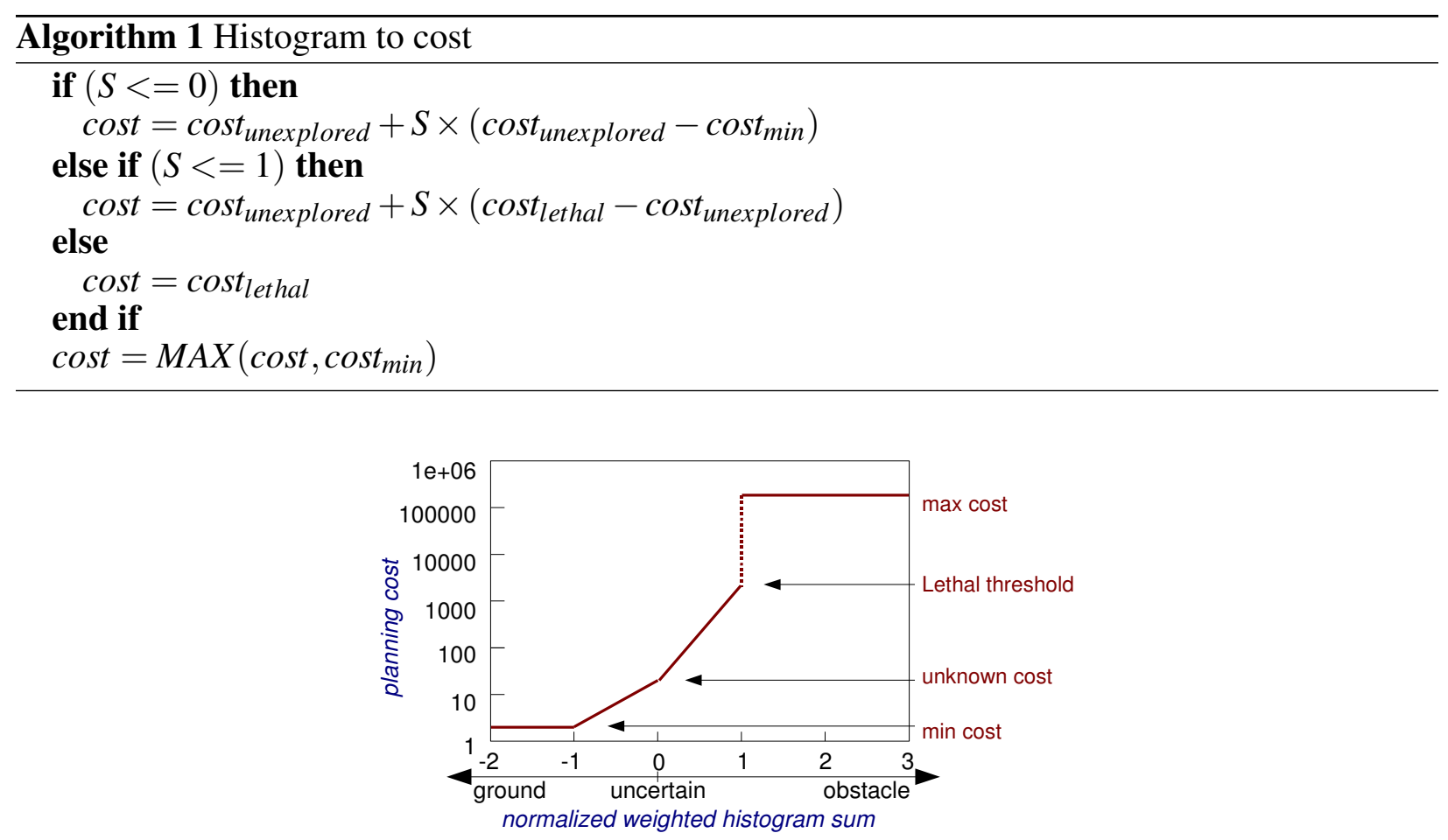

Figure 8: Planning cost function from normalized histogram sum S. An input sum S of 0 represents the full uncertainty, -1 and below are given the minimum ground cost, and 1 or higher the maximum cost.

\subsection{Mapping with Spatial Uncertainty: Hyperbolic Polar Map}

Our map representation is based on two key concepts: 1 . hyperbolic range mapping; 2 . representing categorical evidence by accumulated histograms. In the image plane, a single pixel covers a constant angular extent, but covers a wildly varying range of distances. A single pixel on nearby 
ground (near the bottom of the image) covers a few $\mathrm{cm}$, while a pixel near the horizon covers an essentially infinite range of distances. For a flat ground plane, the mapping of image-plane pixels to distances is hyperbolic from the bottom of the image to the horizon. For this reason, we propose to represent the environment through a robot-centered hyperbolic-polar map (h-polar). This representation allows to map the entire world to a finite number of cells, while being faithful to the type of uncertainty afforded by image-plane labels. To allow the accumulation of evidence for the label of a cell as the robot moves and collects data, each cell in the map contains a histogram of accumulated probabilities for each category. At each frame, the classifier produces likelihood values for each category that are accumulated in the histogram of the corresponding map cell.

Previous work on robot-centered, non-uniform mapping include log-polar representations (Longega et al., 2003) and multi-resolution grid-based maps (Behnke, 2004). The main advantage of the h-polar approach over these methods is the ability to represent an effectively infinite radius with a finite number of cells. Even more important is a representation of range uncertainty that directly corresponds to that associated with image-plane labeling. Pure image-plane labeling and planning (Zhang and Ostrowski, 2002), or visual motion planning, presents the advantage of being free of expensive transformations and pose errors, but can only operate with one single frame at the time. The accumulation of evidence over multiple frames can greatly reduce the perception uncertainty and noise that single frame planning is subject to. In addition, visual motion planning relies on the goal being within the current field-of-view, a dangerous assumption in complicated outdoor scenes. A previous version of our system (Hadsell et al., 2007) used a robot-centered, tactical Cartesian grid mapping with a radius of $30 \mathrm{~m}$. Each time the robot moves and a new camera frame is analyzed, the robot-centered map must be translated and rotated before the results from the new frames are incorporated. This process becomes too expensive for large Cartesian maps. With our new perception system with a range of $100 \mathrm{~m}$, a robot-centered Cartesian map with $20 \mathrm{~cm}$ resolution would have required an impractical 500x500 cells. The h-polar representation offers a considerably more efficient use of memory and CPU.

\subsubsection{Overall Process}

The overall processing sequence for the h-polar map from image labeling to planning is the following:

1. Image labeling (Fig. 9): each pixel of the input images (512x384 in our system) is labeled with an histogram, representing confidences in each class. Each pixel is associated with $(r, c, d)$ coordinates, i.e. row, column and disparity. The disparity (given by stereo) indicates the depth of the point.

2. Plane extraction (Fig. 9): a single plane is found using the short-range stereo points (up to $12 \mathrm{~m})$. Remaining points with no disparity information are projected onto the single plane and given a disparity. $(r, c, d)$ is then converted to vehicle-centered $(x, y, z)$.

3. Point cloud transmission: the point cloud of ( $x, y, z$, histogram $)$ is sent over to the h-polar module.

4. Re-centering memory: Previously accumulated frames are re-centered onto the last available pose.

5. Adding new point cloud: each point of the new point cloud is merged into the h-polar internal point cloud.

6. Produce planning map (Fig. 10): a temporary map for planning and display is produced from the h-polar point cloud. 


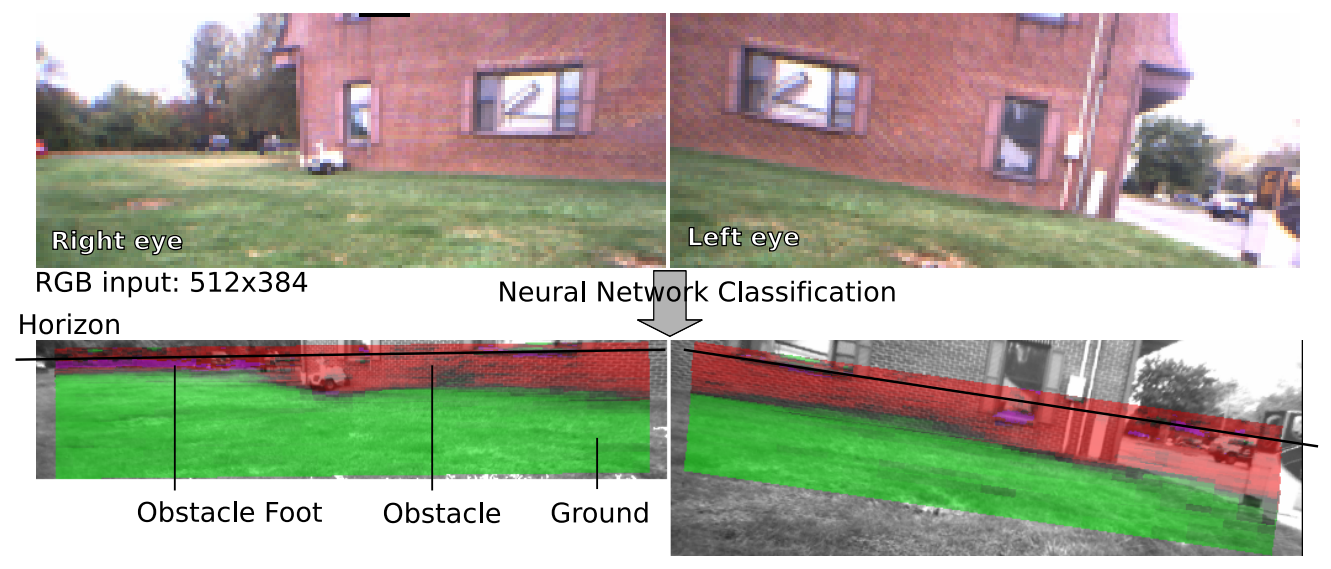

Figure 9: Plane extraction and traversability classification. Left and right images correspond to the left and right camera pairs or "eyes". A single ground plane is extracted from stereo points to generate different vision scales to the horizon, feeding those to the neural network. The resulting classification shows the building and tree line on the left are classified as obstacle (overlaid red) as well as the parking lot on the right (mistakenly). To the contrary, the grass is accurately labeled as traversable (overlaid green). Note: the overlaid colors can only be seen in the color version.

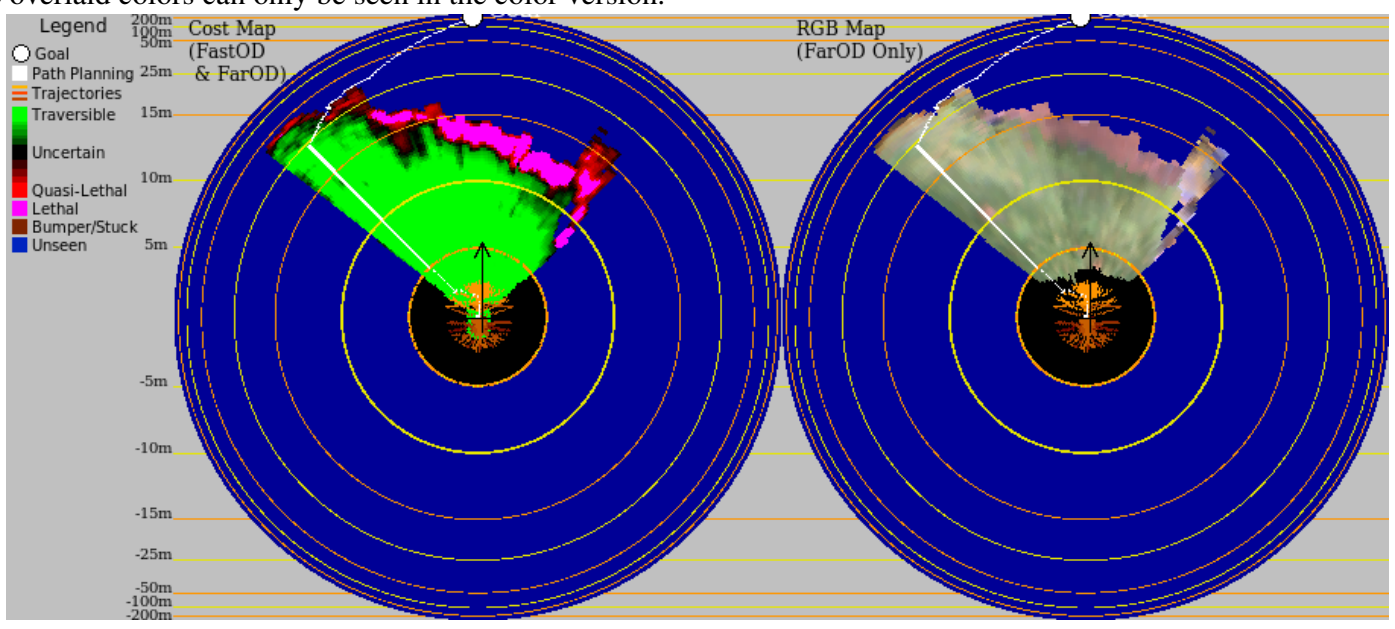

Figure 10: H-polar cost (left) and RGB (right) maps. Those maps are computed from the classification output shown in Fig. 9. The optimal path (in white) to reach the goal 200 meters ahead accurately leads around the building to the left. The RGB h-polar map (right) is used only for display and is very convenient for humans to figure out what the robot is looking at and how good the classification is. The vehicle is alway facing the top of the map. The orange curves in the $2.5 \mathrm{~m}$ radius represent the currently feasible trajectories.

7. Planning: best paths from the goal to all points of the map are computed and sent to the reactive planning layer. Optimal path is shown in white in (Fig. 10).

\subsubsection{Geometry Details}

The h-polar space is not the exact image space given by the real cameras of the robot, but rather a pseudo-image space defined by the pseudo-camera parameters $h_{\text {cam }}$ and $h R_{\min }$ (Fig. 11) and able to integrate multiple frames together. As shown on Fig. 11, the h-polar uses 2 different resolution distributions in the radial dimension:

- From the center of the map to the first hyperbolic cell, the resolution is constant, each cell has the same radial size.

- From the first hyperbolic cell to infinity, the radial cell size increases in an hyperbolic 


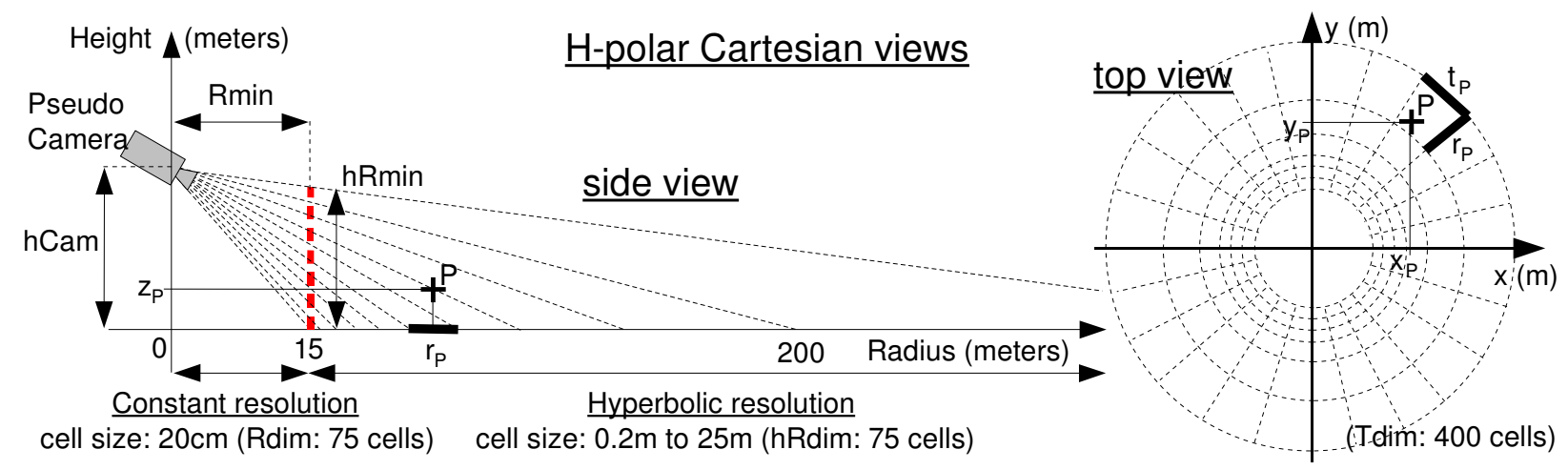

Figure 11: H-polar side and top views. The radial resolution is constant from 0 to 15 meters with $20 \mathrm{~cm}$ radius. Then from $15 \mathrm{~m}$ to $200 \mathrm{~m}$ and more, cell width ranges from $.2 \mathrm{~m}$ to $25 \mathrm{~m}$. Integers $r_{P}$ and $t_{P}$ are the h-polar coordinates of the cell into which Cartesian point $P\left(x_{P}, y_{P}, z_{P}\right)$ falls.

$$
\text { manner (equation of } r_{P} \text { is of type } \frac{1}{\text { radius }} \text { ). }
$$

The radius of the first hyperbolic cell is determined based on the desired cell size $C_{\text {res }}$ in the constant resolution area. In our system, we estimated a cell size of $20 \mathrm{~cm}$ would be sufficient for our purpose. Thus to keep continuity in the cell sizes between the constant and the hyperbolic area, the first hyperbolic cell must begin at a radius $R_{\min }$ of $15.26 \mathrm{~m}$, given the parameters $h_{\text {cam }}$ and $h R_{\min }$ of our pseudo-camera and $h y p R_{d i m}$, the hyperbolic radial dimension. $R_{\min }$ is determined by the following equation:

$$
R_{\text {min }}=C_{\text {res }} \times\left(\frac{h_{\text {cam }} \times h y p R_{\text {dim }}}{h R_{\text {min }}}-1\right)
$$

Following are the values used in our system in the field tests conducted by the LAGR Government

\begin{tabular}{|c|c|c|c|}
\hline$C_{\text {res }}$ & $=$ & $\mathrm{m}$ & constant area. \\
\hline$R_{\min }$ & 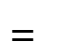 & $26 \mathrm{~m}$ & e first hyperbolic cell. \\
\hline$h_{C a}$ & & $1 \mathrm{~m}$ & Hei \\
\hline & & $0.97 \mathrm{~m}$ & \\
\hline$R_{\text {din }}$ & & 75 & stant area. \\
\hline hypR & $=$ & 75 & ls in the hyperbolic area. \\
\hline$T_{\text {dim }}$ & & 400 & ber of cells in the angular dimensior \\
\hline
\end{tabular}
Team in January 2008:

Given those parameters, Fig. 12 plots the cell index versus the cell radius. Note that cells range up to $350 \mathrm{~m}$. In practice however, because uncertainty increases with distance and because only 150 cells are used in the radial dimension, the system is considered to be accurate up to $100 \mathrm{~m}$. But there are no theoretical boundaries to this limit, which can be increased as computation budget permits.

\subsubsection{Cartesian to H-polar Transformation $(x, y, z)$ to $(r, t, z)$}

Transformation formulas (for the hyperbolic area) from Cartesian coordinates $\left(x_{P}, y_{P}, z_{P}\right)$ in local pose coordinate system to h-polar coordinates $\left(r_{P}, t_{p}, z_{P}\right)\left(z_{P}\right.$ remains unchanged) of point $P$ 

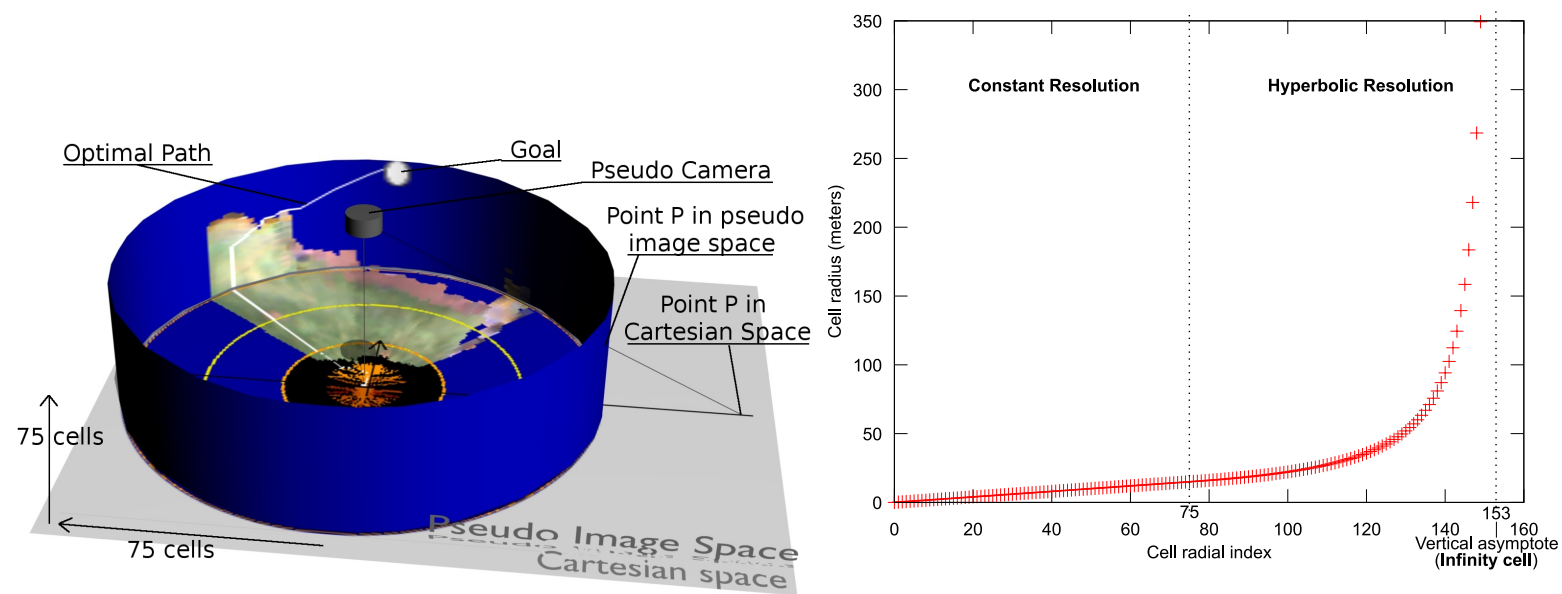

Figure 12: Left: RGB h-polar map in pseudo-image and Cartesian spaces. The center part of the h-polar map has a constant radial resolution whereas the cylindrical part has a hyperbolic radial resolution. When flattened down into Cartesian space, the cylindrical part covers a bigger area in an hyperbolic manner. To transform coordinates between the pseudo-image space and the Cartesian space, a pixel is projected onto the Cartesian space along the line coming from the omniscient camera at the center of the map at height hCam. The reverse transform projects a point from Cartesian space into the pseudo-image space.

Right: h-polar cell radius versus cell radial index with current configuration. Given our pseudo-camera and h-polar parameters, the radial resolution is constant $(20 \mathrm{~cm})$ from cell 0 to 74 with radiuses ranging from 0 to 15.06 meters. From cell 75 to 150 , the resolution is hyperbolic and radiuses range from $15.26 \mathrm{~m}$ to $350 \mathrm{~m}$. Cells sizes range from $.2 \mathrm{~m}$ to $80 \mathrm{~m}$. If few more radial cells were added to our map, infinity would lay in cell 153.

(Fig. 11) are as follow:

$$
\begin{aligned}
& r_{P}=\left(\frac{R_{\text {min }} \times\left(z_{P}-h_{\text {Cam }}\right)}{\sqrt{x_{P}^{2}+y_{P}^{2}}}+h_{\text {Cam }}\right) \times \frac{h y p R_{\text {dim }}}{h R_{\text {min }}}+R_{\text {dim }} \\
& t_{P}=\arctan \left(\frac{y_{P}}{x_{P}}\right) \times \frac{T_{\text {dim }}}{2 \pi}
\end{aligned}
$$

Those transformations are called many times. To remain efficient, their computation must be optimized: pre-computing a look-up table of all the discrete $T_{\text {dim }}$ useful values resulting from the arctan function provides a non-negligible speed-up. Note also that only the integer part of $\left(r_{P}, t_{P}\right)$ is used to determine which cell a point falls into.

\subsubsection{H-Polar to Cartesian Transformation $(r, t, z)$ to $(x, y, z)$}

Transformation formulas (for the hyperbolic area) from h-polar (real) coordinates $\left(r_{P}, t_{p}, z_{P}\right)$ of point $P$ (Fig. 11) to Cartesian coordinates $\left(x_{P}, y_{P}, z_{P}\right)$ in local pose coordinate system $\left(z_{P}\right.$ unchanged) are as follow:

$$
\begin{aligned}
\operatorname{radius}_{P} & =\frac{R_{\text {min }} \times\left(z_{P}-h_{\text {Cam }}\right)}{\left(r_{P}-R_{\text {dim }}\right) \times \frac{h R_{\min }}{h_{y} R_{\text {dim }}}-h_{\text {Cam }}} \\
\alpha & =t_{P} \times \frac{2 \pi}{T_{\text {dim }}} \\
x_{P} & =\cos \alpha \times \text { radius } P_{P} \quad y_{P}=\sin \alpha \times \text { radius }_{P}
\end{aligned}
$$




\subsubsection{Hyperbolic-polar Organized Point-Cloud}

Internally, h-polar is a point cloud, each point being defined by $(x, y, z$, histogram,counter $)$. For practical reasons, the number of points must be bound and it is desirable to keep a uniform distribution of points in the map. Therefore, points are kept using the h-polar distribution, i.e. 1 point per cell, in an array of points of dimensions $\left(\left(R_{\text {dim }}+\right.\right.$ hyp $\left.R_{\text {dim }}\right) \times T_{\text {dim }} \times$ Point $\left._{\text {dim }}\right)$. The dimensions of this map do not have to be the same as the final cost map used for planning. In fact, there are 4 times more cells internally than in this final map. To avoid accumulation of integer imprecisions during re-centering and frame additions, the exact $(x, y, z)$ coordinates are kept at all times. Only the integer part of $(r, t)$ is used to find the target cell of a point.

\subsubsection{Translation and Rotation}

The map is internally fixed onto the local pose coordinate system. It never needs to be rotated except for display. The rotation is extremely simple, a single (ring) offset $t_{\text {offset }}$ is added to the angular index $t$ before access. When re-centering, no rotation is necessary, only translation is applied as described in the following section.

\subsubsection{Re-centering}

Before adding a new frame to the current memory, the latter needs to be re-centered to take into account the movement given by the latest available pose. As pointed out above, re-centering of the h-polar map only requires translation of the $(x, y)$ components of each point:

1. Compute translation $(x, y)_{\text {trans }}$ between the memory's pose and the last known pose in the local pose coordinate system ( $z$ variations are ignored):

$$
(x, y)_{\text {trans }}=(x, y)_{\text {memPose }}-(x, y)_{\text {last Pose }}
$$

2. For all $\left(\left(R_{\text {dim }}+\right.\right.$ hyp $\left.\left.R_{\text {dim }}\right) \times T_{\text {dim }}\right)$ points:

- Add $(x, y)_{\text {trans }}$ to $(x, y)$ components of point.

- Move point into new h-polar cell: if the new cell exists, merge point into that cell, else delete point.

After re-centering, the current angular offset $t_{\text {offset }}$ of the heading of the vehicle $\left(\alpha_{\text {last Pose }}\right)$ must be saved in order to render a map fixed to the vehicle coordinate system:

$$
t_{\text {offset }}=\alpha_{\text {lastPose }} \times \frac{T_{\text {dim }}}{2 \pi}
$$

Later, $t_{\text {offset }}$ simply needs to be added to each $t$ index before accessing the cells for render.

\subsubsection{Adding a New Frame}

A new frame comes as a cloud of ( $x, y, z$, histogram) points centered on the vehicle's coordinate system at the time of the input image capture. Before adding new points to the memory (already re-centered with last known pose), translation and rotation need to be applied to take into account the last known position of the robot:

$$
(x, y)_{\text {trans }}=(x, y)_{\text {imgPose }}-(x, y)_{\text {lastPose }}
$$


The rotation corresponds to the heading $\alpha_{i m g P o s e}$ of the robot in the local pose coordinate system, given by imgPose, the pose of the image. Hence the following transformation matrix ( $\mathrm{z}$ remains unchanged and can be ignored) produces the centered coordinates $(x, y)^{\prime}$ :

$$
\left[\begin{array}{l}
x \\
y
\end{array}\right]^{\prime}=\left[\begin{array}{ccc}
\cos \alpha & -\sin \alpha & x_{\text {trans }} \\
\sin \alpha & \cos \alpha & y_{\text {trans }}
\end{array}\right] \cdot\left[\begin{array}{l}
x \\
y \\
1
\end{array}\right]
$$

To add the re-centered new frame to the re-centered memory, one simply need to loop through all points of the image point cloud and merge each into their corresponding h-polar cell.

\subsubsection{Points Merging}

When re-centering memory or adding a new frame, several points may fall into a same h-polar cell and need to merge together since only one point per cell is kept. There are two different merging operations to consider:

- Data merging: Using the histogram scheme, merging is a simple addition. Keeping a counter of the number of points merged can be useful to normalize the RGB display and for coordinates merging.

- Coordinates merging: The new coordinates can be an interpolation of each point's coordinates weighted by their counter, thus giving more importance to points seen many times. However in our system, because of a low computation budget, coordinates are simply overwritten by the last added point.

\subsubsection{Blocking after Obstacles}

A key element to avoid projecting down to the ground the part of obstacles for which no $z$ estimate is available, typically points beyond stereo range, is to block those points after an obstacle. For example, a tree trunk can appear as a giant obstacle in the map if not blocked after its foot. Therefore, all points without $z$ information following an obstacle in the radial direction are ignored when adding a new frame.

\subsubsection{Sub-sampled and Smoothed Mapping for Planning}

The internal high resolution h-polar map is scattered because points can merge during the translation process. This can produce erratic path planning between unknown cells and create holes inside lethal obstacles. To cope with this issue, a lower resolution map is produced for the actual planning: every low-resolution cell is a sum of 4 neighboring cells. In addition to sub-sampling, as some holes may subsist, the 8 neighbors of each cells are added, thus providing a smooth dense map. Note that no normalization is required since the histogram scheme handles addition of multiple histograms by modulation of confidence.

\subsubsection{Registration for Increased Accuracy}

To improve mapping accuracy, registration methods can be used before merging new frames into the h-polar memory map. The pseudo-image space provides an easier and more efficient space for patches registration to correct for pose imprecisions. Registration was not implemented in the current system but should be investigated in further research. 


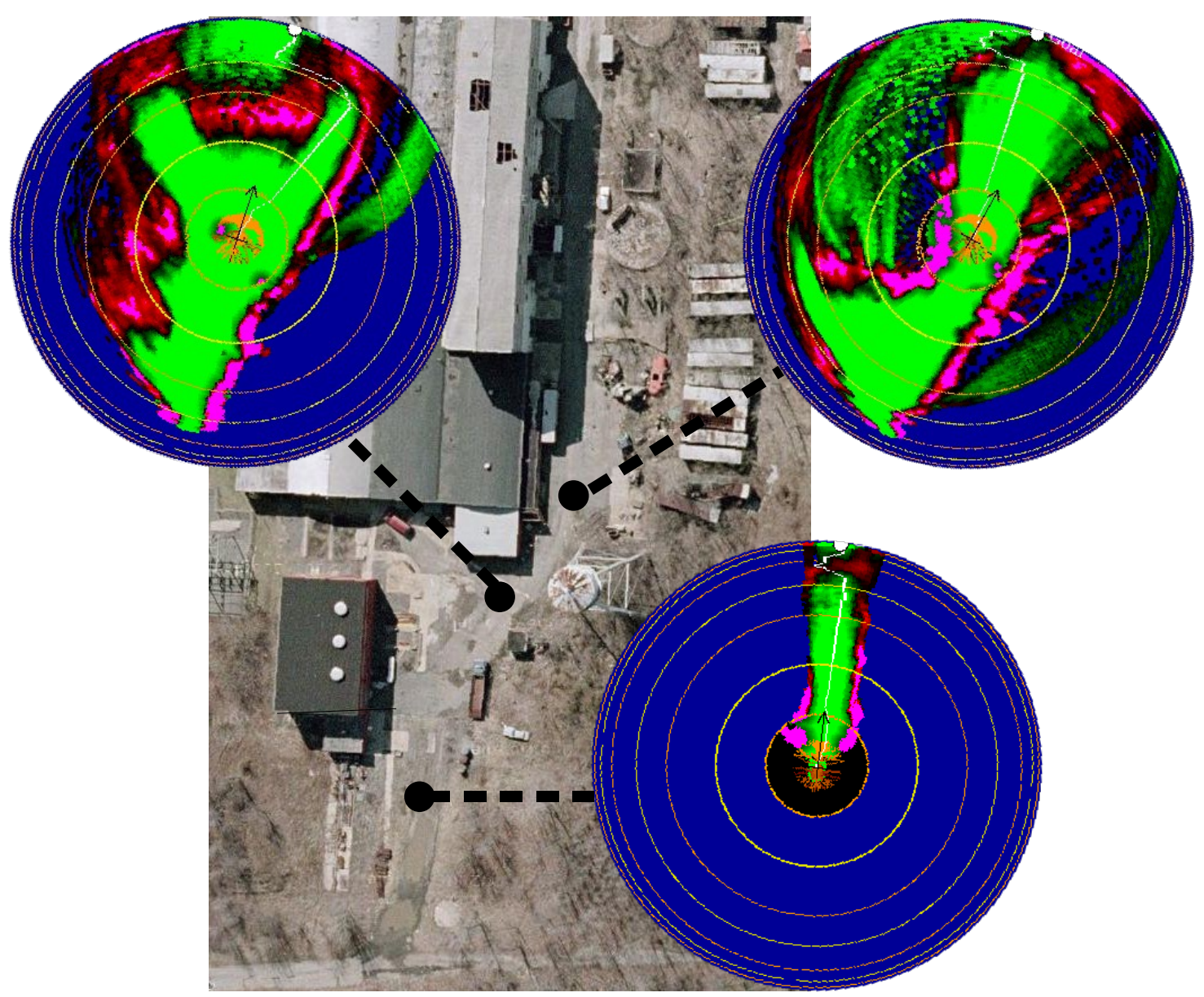

Figure 13: h-polar maps along heterogeneous outdoor course. The robot starts at the bottom of this satellite image going to the top. Each h-polar map accurately represents the real environment and plans around obstacles from an approximate distance of 50 meters.

\subsubsection{Grid-based Planning with Pre-computed Transition Costs}

Any grid-based planning algorithm can be used here by using the pre-computed transitions costs between the center of each cell to their 8 neighbors (the transition cost is simply the Euclidean distance of the $(x, y)$ real coordinates of each cell's center). As described earlier, the multi-layer planning is used to guarantee latency independency between processes. Thus the hyperbolic-polar planning must generate all best paths from the goal to all cells in order to allow the lower planning level to operate on its own for few iterations at a higher frame-rate. We use the Dijkstra algorithm from the goal to every cell of the h-polar map as our single-source to multi-targets planner. Every h-polar cell contains the optimal path to the goal. The reactive planner computes the cost from the vehicle center to a finite number of angular candidates at a certain radius $(5 \mathrm{~m})$, then queries and add the remaining cost for each of those candidates, beforehand translated into h-polar coordinates. The candidate with minimum cost contains the optimal path. Finally, the list of wheel commands of the trajectory that initiated the best path is executed until the next iteration. The optimal paths are drawn in white in Figs. 13 and 14. 

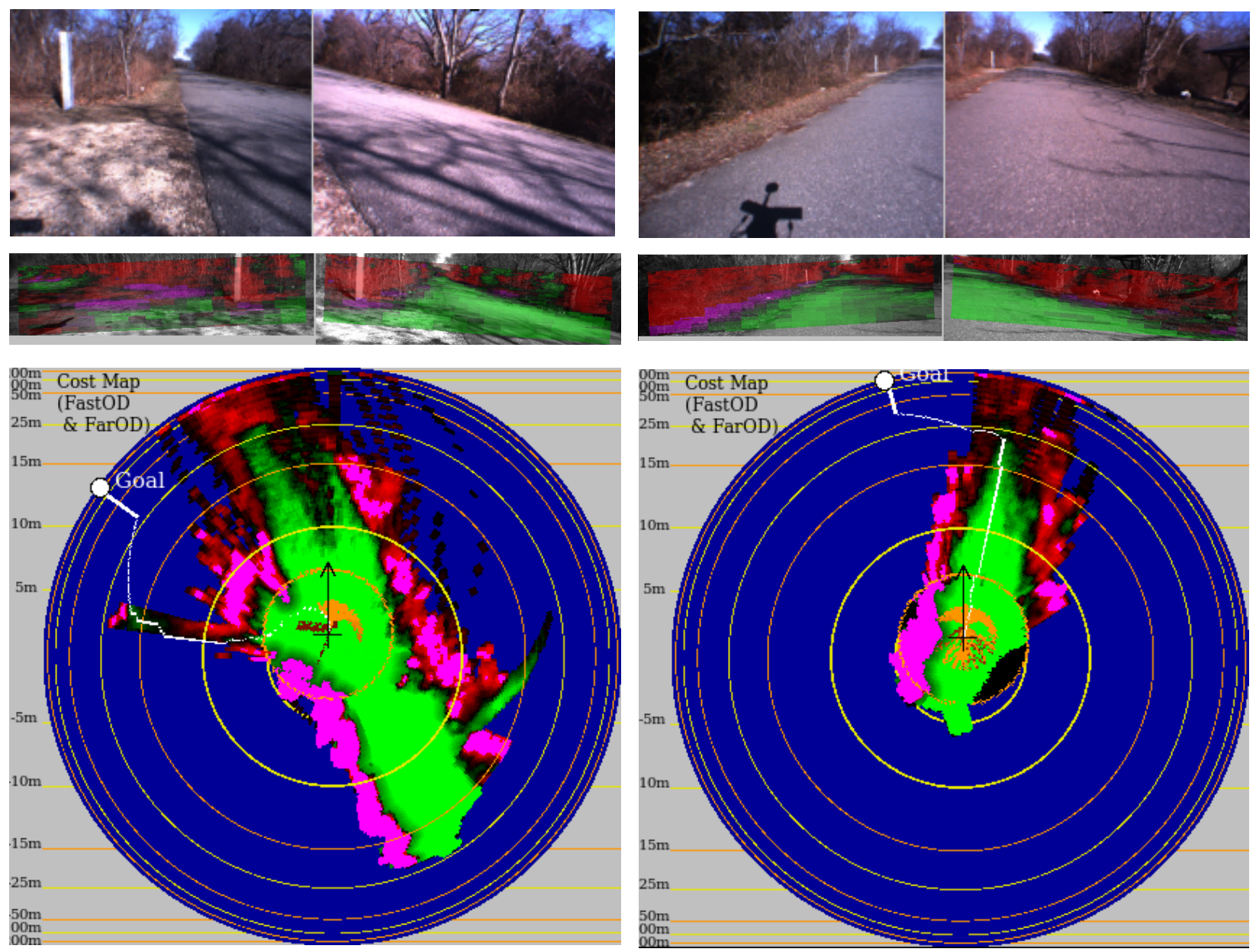

Figure 14: Result runs with long-range vision activated in Sandy Hook. The top images are left and right input images. The middle images show classification for left and right. At the bottom is the corresponding h-polar map. Note: the classification images in the middle for left and right examples were taken a few frames later compared to the input and the h-polar images.

\section{Layer 1: Fast Perception and Planning}

\subsection{Fast Stereo Vision}

The short-range module (in Layer 1) follows a standard approach to vision-based obstacle detection (for similar examples, see (Kelly and Stentz, 1998; Kriegman et al., 1989; Goldberg et al., 2002)). But it is also meant to process as fast as possible to guarantee a low-latency response to obstacles in the short-range as part of the navigation architecture. Results in Tables 1 and 2 have shown that using a resolution as low as $160 \times 120$ for the stereo vision does not entail collisions with the LAGR vehicle. By decreasing the resolution, we also decrease the vision range $(5 \mathrm{~m})$ but we increase reactivity. From those results we conclude that reactivity prevails over vision range in the short-range.

Along with the low-resolution, simple heuristics are used to produce traversability labels from the stereo 3D points. A single ground plane fitted to the points is first estimated using a Hough transform as described in (Hadsell et al., 2008). Points are then projected onto that ground plane and separated into the cells (10 $\mathrm{cm}$ squares) of the Cartesian map $(10 \mathrm{mx} 10 \mathrm{~m})$. The number of points 
falling into each cell is tallied and a threshold determines if a cell is an obstacle or traversable (unknown if empty). This small Cartesian map of 100x100 cells is then fed to the trajectory planning module described next. This small local map is located at Layer 1 but is also sent up to Layer 2 to be merged into memory in order to increase confidence in the h-polar map, and also reduce disagreements between Layer 1 and 2 maps. If Layer 1 sees an obstacle that Layer 2 does not, the robot can go in loops forever in front of a cul-de-sac: Layer 2 sees an opening in the culde-sac and leads the robot in it, but getting closer Layer 1 realizes it is actually closed, ordering to go away from it. But going away from it, Layer 2 will bring the robot back when out of the Layer 1 range.
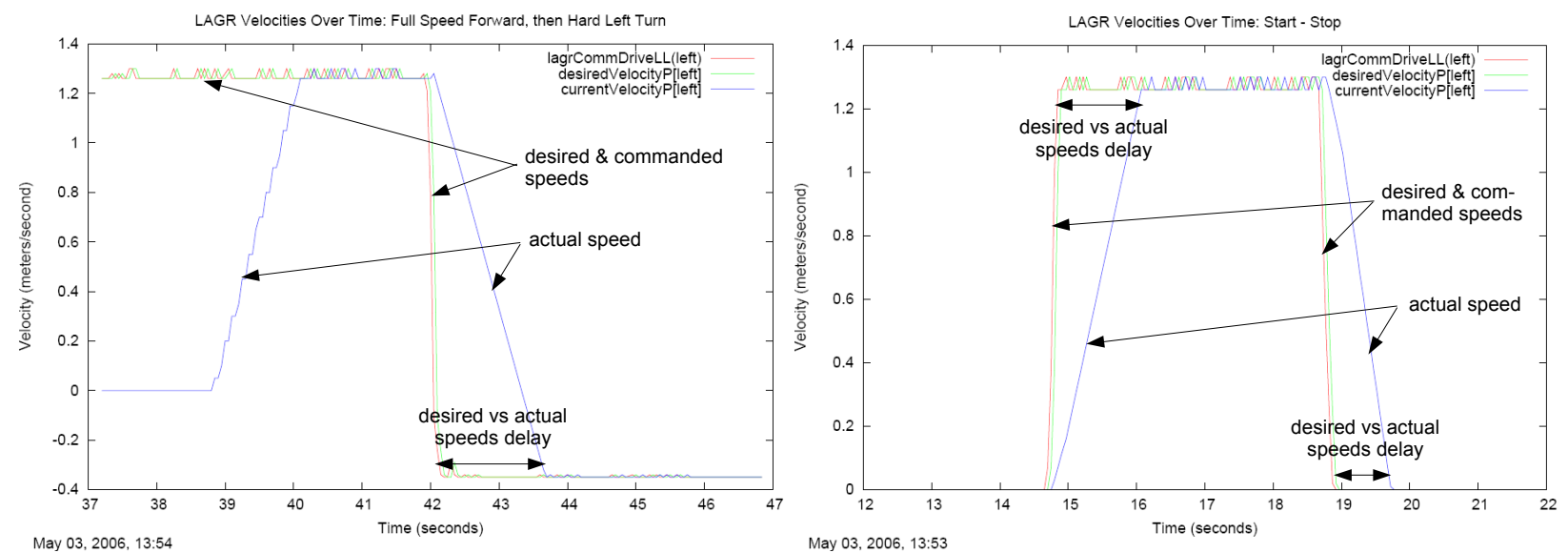

Figure 15: To illustrate the dynamics of the LAGR vehicle $(109 \mathrm{~kg}$ ), the plots show the desired speed (green and red) of the left wheel versus its actual speed (blue). In the left plot, the robot is driven full speed forward followed by a hard left turn. It takes the left wheel about 1.6 seconds to go from the full forward speed of $1.3 \mathrm{~m} / \mathrm{s}$ to the full backward speed of $-0.35 \mathrm{~m} / \mathrm{s}$. Similarly in the right plot, the robot is driven from $0 \mathrm{~m} / \mathrm{s}$ to full speed forward then back to $0 \mathrm{~m} / \mathrm{s}$. It takes the robot about a second and half to reach the full speed and about a second to stop.

\subsection{Fast Dynamics Planning: Recorded Maneuvers}

An essential module for collision-free navigation is a fast reactive planner incorporating vehicle dynamics. The simple and computationally fast method described below is able to record sophisticated dynamics models while avoiding the complex and potentially inaccurate modelling of traditional methods.

Vehicle dynamics is typically handled by models whose parameters are found through system identification or manually computed from the vehicle's characteristics. While these methods provide accurate theoretical dynamical models, they may not take into account differences between individual vehicles, lack adaptability to new environments and may not handle sophisticated models, requiring hand-crafted heuristics for backwards motion for example. We propose a simple and computationally efficient method that bypasses the need for system identification or hand-crafting heuristics. Our system learns the particularities of individual vehicles, allows on-line adaptation and sophisticated models.

Human-driven or autonomously-driven trajectories are recorded and stored. While in learning mode the robot records each traveled trajectory and attempts to place it into a bank indexed by the initial speeds of each left and right wheels and the ending position of the trajectory at a fixed radial distance from the current (starting) position. Only the best trajectories (mainly the fastest ones, other measures are described further) are stored in the trajectory bank. These are reused (essentially played back) during autonomous runs for precise vehicle control. The system knows 
the state in which it starts and has a recorded set of wheel commands from that state to any point on a circle of a chosen radius.

Optimal control techniques (Bryson and Ho, 1975) for motion planning provide optimal solutions but suffer from high computational costs, making them impractical for real-time applications. Most efforts have concentrated on curve-fitting for online planning from line segments (Tsumura et al., 1981) or arcs (Komoriya et al., 1984) or clothoids (Kanayama and Miyake, 1985) or cubic spirals (Kanayama and Hartman, 1988). Curve-fitting relies on identification of the parameters of a vehicle's dynamical model. Our method provides a simple and automatic identification of model parameters while bypassing the complex curve-fitting problem and resulting in a nearly null online computation cost. (PiedMonte and Feron, 1999) introduced maneuver-based planning in free environments for aerial vehicles by recording human experts maneuvers, and later developed a maneuver-based automaton (Frazzoli et al., 2005) that can concatenate trajectory primitives with a regular language. While the concept of trajectory recording for maneuver-based planning is the same, we extended it to perform obstacle avoidance as well, and apply it for ground robots non-holonomic planning. A similar pre-computed dynamics planning architecture was introduced by (Coombs et al., 2000) but using an off-line clothoids pre-computation method which still relies on hand-crafted modelling. (Kelly and Nagy, 2003) points out the need for a parametric trajectory representation in contrast to the prohibitively large space required by pre-storing methods. The practical results of (Coombs et al., 2000) and this paper advocate in favor of trajectory pre-storing. While recording and pre-storing can certainly not address all non-holonomic planning problems, its simplicity and efficiency make it worth considering for some systems. Our recording method is currently limited to 2D planning and does not address the 3D challenges treated in (Spenko et al., 2006; Howard and Kelly, 2005).

\subsubsection{Recording Trajectories}

Trajectories can be recorded every time the robot moves, either in supervised mode (human driver) or self-supervised (autonomous). On the LAGR platform, information about the state of the vehicle is available at $20 \mathrm{~Hz}$. This state includes among other information an absolute time-stamp, the current pose and current speeds of each wheel. Similarly, wheel commands are sent at $20 \mathrm{~Hz}$ to the motors in either manual or autonomous mode. These selected state variables along with the wheel commands are grouped into a sample every $50 \mathrm{~ms}(20 \mathrm{~Hz})$. By recording this stream of samples, a feasible trajectory reaching the current position and the corresponding series of wheel commands are known at each time-stamp. In other words, the radius $R_{t r a j}$ to the current vehicle center can be reached by looking back in the recorded sample stream and executing the wheel commands found in the stream. Every 50ms a new trajectory is produced and after some driving, enough trajectories are recorded to reach radius $R_{t r a j}$ in all directions. The trajectory space has other input dimensions: the initial state $\left(\right.$ LSpeed $_{0}$, RSpeed $\left._{0}\right)$. Composed of both wheel speeds obtained from the vehicle state, the initial state allows the system to select the current set of feasible trajectories. When disregarding terrain differences (ice vs. asphalt), left and right initial wheel speeds are the main variables determining the current dynamics. For example in Fig. 6, initial state $(4,9)$ means that the robot is making a hard left turn at $t=0$. By analyzing the trajectories going to the right, the figure makes clear that the dynamics of the vehicle have been captured. From this start state (turning hard left) in order for the vehicle to reach a candidate 90 degrees to the right it must make a large loop where left-turning and forward moving momentum is transformed into a right turn. This curve has not been computed, but simply selected. It is the fastest set of commands seen so far for getting the vehicle from a hard left turn to a hard right.

Of course the accuracy of the trajectories depends on the accuracy of the pose sensor, which in the LAGR platform relies on a combination of the wheel odometers and an IMU. This pose information 
is assumed to stay accurate enough in the short term. If no visual odometry correction is available, it is preferable to record trajectories only on surfaces with low pose error rate (i.e. asphalt is preferable to ice). Once trajectories are extracted from the sample streams, they need to be sorted, compared and stored into the trajectory bank (pre-storing).

\subsubsection{Trajectory Bank}

The trajectory bank holds all best recorded trajectories. Each trajectory is indexed by $\left(\right.$ LSpeed $_{0}$, RSpeed $_{0}$, Angle $\left._{R_{\text {traj }}}\right)$. LSpeed 0 and RSpeed $_{0}$ are the left and right speeds at time 0 of each trajectory. The LAGR vehicle drives at speeds ranging from $-0.5 \mathrm{~m} / \mathrm{s}$ to $1.3 \mathrm{~m} / \mathrm{s}$. This range is quantized into 10 different bins $(0 \mathrm{~m} / \mathrm{s}$ lies in index 3$)$. There are thus 100 different possible initial speed states. For each speed state, 160 Angle $_{R_{t r a j}}$ candidates are evenly divided around the perimeter at $R_{\text {traj }}$ radius. In Fig. 6, speed states along the diagonal have similar left and right wheel speeds at $t_{0}$, whereas at the top right and bottom left, the robot is steering harder right and left respectively. Because very hard turns are less frequent, these corners are more sparse than the area around the diagonal. A minimal bank with only $15 \%$ of all states filled (extracted from approximately 2 hours of human-driven recorded samples) showed great driving performance as demonstrated in Table 2. The bank shown in Fig. 6 is $64 \%$ full and was obtained using 18 hours of human-driven and autonomous-driven log-files (recorded during regular testing runs). The latter bank shows similar performance as in the Table 2 but can deal with a wider range of situations.

The discretization of wheel speeds and angular candidates as well as the radial distance of the trajectories does require some tuning based on the maximum driving speed of the vehicle and the available computational budget. The higher the maximum speed of the vehicle, the more wheel speed bins are required and the more the trajectory radius must be increased. With more resolution (more wheel states) in each input dimension, modeling accuracy increases but so do computational requirements. Moreover, the more states in the bank, the more trajectories need to be recorded. It was empirically found that on the LAGR platform, a minimum of 5 states per wheel was sufficient to obtain decent driving. 10 states provided the best results while keeping a rather small bank. The required number of angular candidates is estimated from the local map's resolution. With a cell size of $10 \mathrm{~cm}$ and a radius of $2.5 \mathrm{~m}$, approximately 160 candidates $(2 \pi \times 25)$ are needed to have 1 trajectory per cell on the perimeter. The $2.5 \mathrm{~m}$ radius was also chosen empirically and is based on the time the vehicle can travel before an update to the maps from the planner. Is it better to keep the radius as small as possible to limit the trajectory sampling space for memory and bank-filling matters.

Since in a race the vehicle is expected to minimize traveling time, the simplest scoring criteria for trajectory selection is the time it takes to reach the radius $R_{t r a j}$. When multiple trajectories are available for a same candidate, only the one with the lowest score is kept. Different scoring formulas yielding additional trajectory types for increased navigation performance are described in further sections.

\subsubsection{Planning with Trajectories}

The first step in planning is to determine the current speed state from the motor sensors, i.e. the speed of each wheel. This speed state indexes from the bank the set of currently feasible trajectories (based on what we have recorded). Next, the set of trajectories is tested against the current costmap as shown in Fig. 6. All trajectories passing through non-traversable cells are ignored, the remaining set of possible trajectories are assigned a cost traj based on the cost of the traversed cells in the map and the recorded traveling time of the trajectory time $_{\text {traj. }}$. Cell costs represent 
traversability difficulty in seconds per meter, the minimum cost cost $_{\min }$ of perfectly traversable terrain is $0.77 \mathrm{~s} / \mathrm{m}$ (vehicle's maximum speed is $1.3 \mathrm{~m} / \mathrm{s}$ ). Assuming trajectories are recorded on easy ground (e.g. asphalt), the minimum cost traj equals the sum of $t_{i m e} e_{t r a j}$ and an "extra" cost given by the cells as follows:

$$
\operatorname{cost}_{t r a j}=\text { time }_{\text {traj }}+\sum_{i}^{n_{\text {cells }}}\left(\operatorname{cost}_{\text {cell }_{i}}-\operatorname{cost}_{\text {min }}\right)
$$

This formula augments the real recorded time of the trajectory by adding extra time based on the estimated difficulty of traversing a cell whose terrain has been classified by the FarOD and FastOD map creation.

Trajectory costs are available on the $2.5 \mathrm{~m}$ radius around the robot. From those costs, costs of optimal paths to each cell on the $5 \mathrm{~m}$ radius are computed using the Dijkstra algorithm. The costs on the $5 \mathrm{~m}$ radius are added to the Layer 2 planning costs to form a global cost. The trajectory minimizing the global cost is selected and sent to the lower level planning execution process (Layer 0 ), thus protecting lower levels from latencies in more complex higher levels. The top planning level (Layer 2), running at $1 \mathrm{~Hz}$ using the Dijsktra algorithm computes all optimal paths from the goal to each cell of the map and passes it on to Layer 1 which runs at 5 to $10 \mathrm{~Hz}$. Computing paths to all cells of the map is necessary because the Layer-1 map moves relative to the Layer- 2 map, and thus uses different interfacing cells for every Layer-1 iteration. Once the best trajectory is selected, a list of wheel commands is sent to the Layer-0 process which is responsible for sending wheel commands at $20 \mathrm{~Hz}$. The resulting multi-layer mapping and planning is represented in Fig. 5.

\subsubsection{Tail whacking}

To reduce computation, the width of the robot is taken into account by growing obstacles in the map by half the vehicle width. This simple method is fast but assumes that the length of the robot is null. Unaware of its length, the robot would often whack obstacles with its tail while turning. This issue is easily resolved by adding and keeping track of one or more points along the robot's length axis Fig. 16. A trajectory is discarded during planning if either the head or the tail trajectories encounter a non-traversable cell. With only one additional point near the tail of the LAGR robot, tail whacking completely disappeared during the numerous field tests.
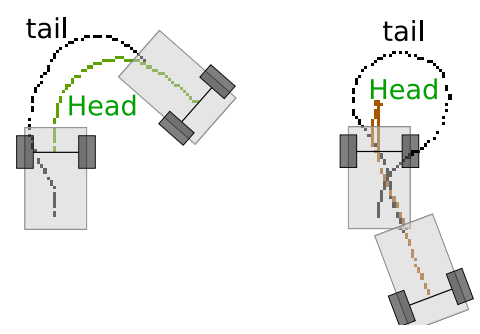

Figure 16: Tail whacking. To account for the vehicle's length and to avoid hitting obstacles with the tail, one additional "tail" trajectory is computed from each recorded "head" trajectory. (Width is handled by obstacle growing.) During planning, a trajectory is ignored if either the head or the tail of a trajectory encounters a lethal cell. Multiple tail trajectories can be added depending on the vehicle's length, but only one tail trajectory was necessary to get rid of the tail whacking issue on the LAGR vehicle. 


\subsubsection{Fail Safe}

The vehicle may fall into a state that has no recorded trajectory if it reaches a rare state (very hard turns), if the bank is not full enough or if the vehicle is completely boxed-in by obstacles. A simple solution is to stop the robot if it is moving or turning to let it fall into another state where there will be a useable trajectory. If it reaches the null speeds state and still no trajectory is available then, a backup mode is triggered (scripted driving straight backwards with a slight turn) to move the vehicle away from obstacles until feasible trajectories are available again. This simple solution has proved to be efficient as the vehicle is never stuck standing still nor stuck in backup mode in any of the numerous field tests.

\subsubsection{Refinements}

The simple recording solution described above required minimal engineering and tuning efforts while providing a computationally cheap and collision-free navigation platform. Following are few additions to the system which brought further improvements. Other additions that increase the robot's self adaptability to changing environments are described in the future work section.

When planning with obstacles having only one trajectory per initial wheel state and angular candidate can be restrictive. A single obstacle close to the vehicle can wipe out all the trajectories in a general direction. To give more opportunities to the planner it is desirable to store multiple trajectories taking different paths to the same candidate. Storing multiple banks each with different criteria for selecting the trajectories one can achieve this goal. For example, one might record trajectories that take as straight a path as possible to the candidate, as well as trajectories which steer to the left or right before taking the fastest path to the candidate. This allows the planner to pick a trajectory which goes around an obstacle on its way towards the optimal candidate for planning in the larger map.

By sampling only those trajectories which drive fastest from where the vehicle is to the 160 angular candidates around it, we are selecting the behavior we want from the vehicle (to drive fast) but also greatly reducing the space of all possible vehicle movements (all sequences of wheel commands) and other desirable behaviors. By adding other selection criteria we can more completely cover the space of possible movements, and produce more complex behaviors. For example in some situations, such as when the robot has made a wrong turn and gotten boxed in, it is desirable for the system to stop, turn in place and go straight rather than keeping momentum by driving as fast as possible along smooth curves. These different types of behaviors can be obtained simply by using different scoring measures when selecting trajectories from the recorded samples for a bank.

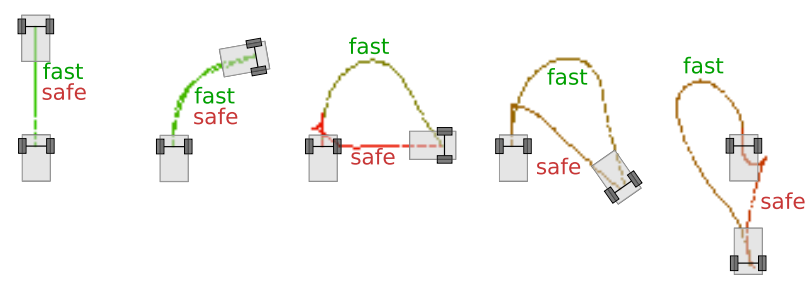

Figure 17: Fast and safe trajectories: few examples of different trajectories obtained for a same end point using different scoring measures. The measure fast which rewards fastest trajectories, selected smooth trajectories with large curves, maximizing the vehicle's speed. The measure safe rewards maximum initial alignment of the vehicle's heading and the candidate heading and thus selected trajectories which cause the vehicle to stop and turn in place before driving straight to the candidate. Having dissimilar trajectories for a same candidate helps the vehicle to properly handle different situations.

Two types of trajectories were extensively tested and called fast and safe trajectories. The fast 
trajectories are selected by minimizing the time to reach the $2.5 \mathrm{~m}$ radius and maximizing the distance traveled in the first few samples as follows. For all trajectories filling a single slot in the bank $\left(\right.$ LSpeed $_{0}$, RSpeed $_{0}$, Angle $\left._{R_{\text {traj }}}\right)$, retain the one that minimizes:

$$
\text { time }_{\text {traj }}-\sum_{k=0}^{n_{\text {cmds }}-1}|k, k-1| \times \text { decay }{ }^{k} \times \gamma_{\text {dist }}
$$

where $n_{c m d s}$ is the number of wheel commands of the trajectory and $|k, k-1|$ the Euclidean distance between two samples, measured by the vehicles wheel encoders. $\gamma_{\text {dist }}$ is the normalization term between the time measure and the distance measure. By using the exponentiation of decay (Fig. 18), we ensure that only the distance at the beginning is maximized. Because the fast planner picks a new trajectory every $100-250 \mathrm{~ms}$, in practice only the first few commands of a trajectory are actually driven therefore it is important to have a term which forces the desired behavior to happen at the beginning of the trajectory, hence the exponential decay terms.

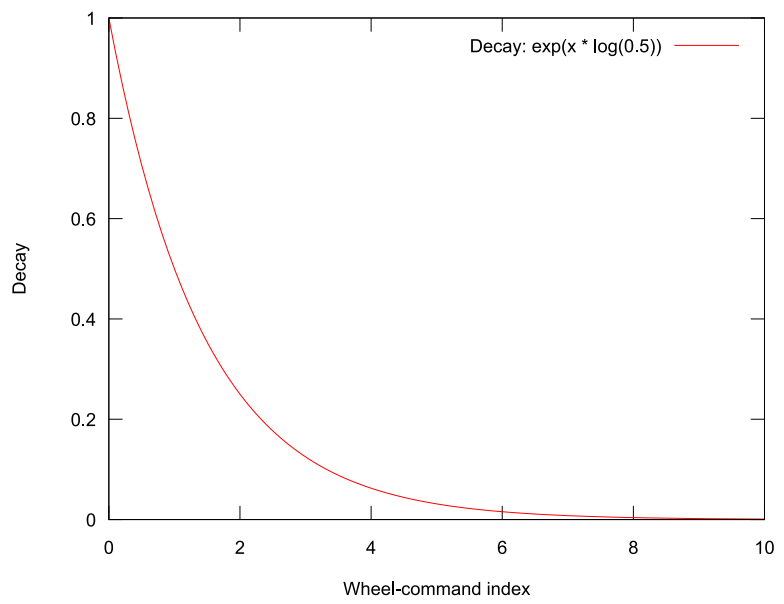

Figure 18: Trajectories types decays: a decay of 0.5 is exponentiated with the wheel-command index in order to give more importance to first samples and near no importance after 5 samples for the distance and heading measure of the fast and safe trajectories.

Safe trajectories, on the other hand try to minimize the traveled distance and the angular difference between the vehicle's heading and the candidates heading, thus forcing the vehicle to drive as closely as possible to the ray from the vehicle to the candidate. To achieve this, the scoring formula minimizes the traveled distance while maximizing the initial turning toward the target heading angle $_{\text {cand }}$. The resulting behavior is turn on a dime and go straight in the candidate's direction. For all trajectories reaching a single $\left(\right.$ LSpeed $_{0}$, RSpeed $_{0}$, Angle $\left._{R_{\text {traj }}}\right)$, retain the one that minimizes:

$$
\sum_{k=0}^{n_{\text {cmds }}-1}|k, k-1|-\mid \text { angle }_{\text {cand }}-\text { angle }_{k} \mid \times \text { decay }^{k} \times \gamma_{\text {heading }}
$$

where angle $_{k}$ is the heading of sample k. As in the fast trajectories, decay (Fig. 18) helps maximize turning in the first few commands. $\gamma_{\text {heading }}$ is the normalization term between the distance measure and the heading measure.

\subsection{Fast Rotational Visual Odometry}

The ability for a robot to localize itself can be critical for successful autonomous operation. While a globally consistent solution to the localization problem must necessarily also perform mapping 
(Thrun, 2003), many applications do not require or benefit from a globally consistent map. Locally consistent approaches such as fixed-time-window SLAM (Bibby and Reid, 2007) and visual odometry (Nistér et al., 2004; Agrawal and Konolige, 2007) have shown great success in applications such as goal-directed navigation and localization in dynamic environments.

Wheel odometry has been a popular mainstay for robotic localization due to its low overhead and high sampling frequency. Its accuracy however is limited by wheel slip, a source of error that can be challenging to detect and correct without other sensors. Wheel slip can be particularly frequent and destructive in runs over outdoor terrain with loose or uneven ground. Full visual odometry (VO) uses feature tracking to entirely replace ground odometry, but current systems (Agrawal and Konolige, 2007) require 100\% of the processing time on a high-end CPU. For singleCPU autonomous robots, this cost can be prohibitive. The cost of VO can be a burden even for multi-CPU platforms, as it is often desirable for all camera-computer pairs to be able to perform additional tasks at high frame-rates, such as short-range obstacle detection.

We have implemented a visual localization system that runs at a fraction of the cost of state-of-theart VO systems while maintaining comparable accuracy. On our multi-processor, multi-camera system, this allows a single processor-camera pair to handle VO in parallel with other visual tasks, enabling tight coupling between localization, obstacle detection, and control. Our system can be of even more use to robots with limited computational power, such as small robots, consumer-oriented platforms, or extraterrestrial rovers.

We achieve this performance gain by specializing the visual odometer to the task of tracking only one degree of freedom: the robot's bearing. This bearing estimate is combined with a wheel odometer's translation estimate to yield 3-DOF pose estimates with comparable accuracy to stateof-the-art 6-DOF visual odometers. The efficiency of our system comes from the fact that tracking only the bearing allows us to operate at much lower resolutions than would be acceptable on a 6DOF odometer. This is because the uncertainty of an object's distance grows rapidly with distance at low resolutions, while the uncertainty of its robot-relative bearing is constant. For example, at our resolution of $160 \times 120$ pixels, an uncertainty of \pm 0.25 pixels translates to \pm 0.1 degrees of yaw, but \pm 1.25 meters of distance for an object 6 meters away. Additional speedups are gained by using spherical image projection for more reliable feature tracking, and limiting the feature tracking to windows bounded by wheel odometer output. We show that this hybrid odometry approach achieves much of the benefit of a full visual odometer at a greatly reduced computational cost.

\subsubsection{Related Work}

There has been much work in both wheel odometry and visual odometry (VO), while only limited attention has been paid to the intersection of the two approaches. (Schäfer et al., 2007) use wheel odometry to check the output of a full VO system for errors arising from moving objects. Rather than run full visual and wheel odometry systems in parallel, we have focused on how to best exploit wheel odometry to lighten the computational burden of VO. A number of authors have used visual matching to correct IMU or GPS data on aerial platforms (Brown and Sullivan, 2002) (Andersen and Taylor, 2007) (Veth et al., 2006). Our system is implemented on a ground rover, where many of the assumptions afforded in the air, such as nearly coplanar features and slow visual flow, do not apply. (Veth and Raquet, 2006) use IMU output to guide the feature search of a groundbased visual odometer, running on a manned sensor platform that takes pictures at $1 \mathrm{~Hz}$. Many autonomous vehicles require a higher frame-rate to ensure overlapping features between frames, and to ensure that spurious poses from the IMU or wheel odometer are corrected before the robot controller reacts. 


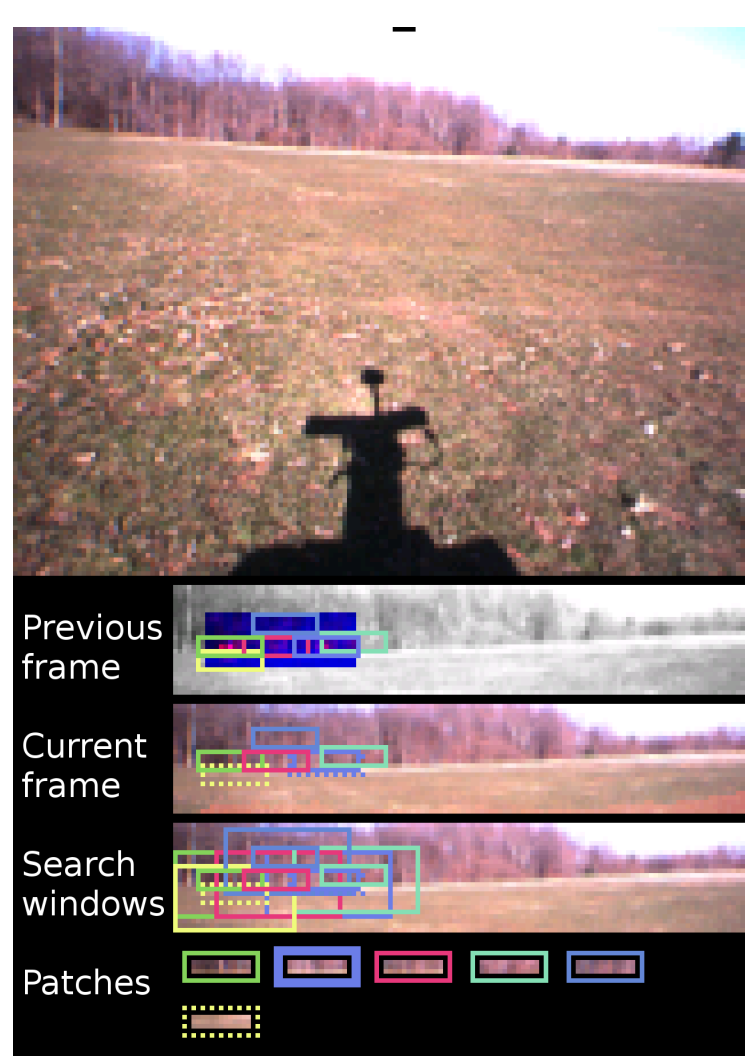

(a) Open field

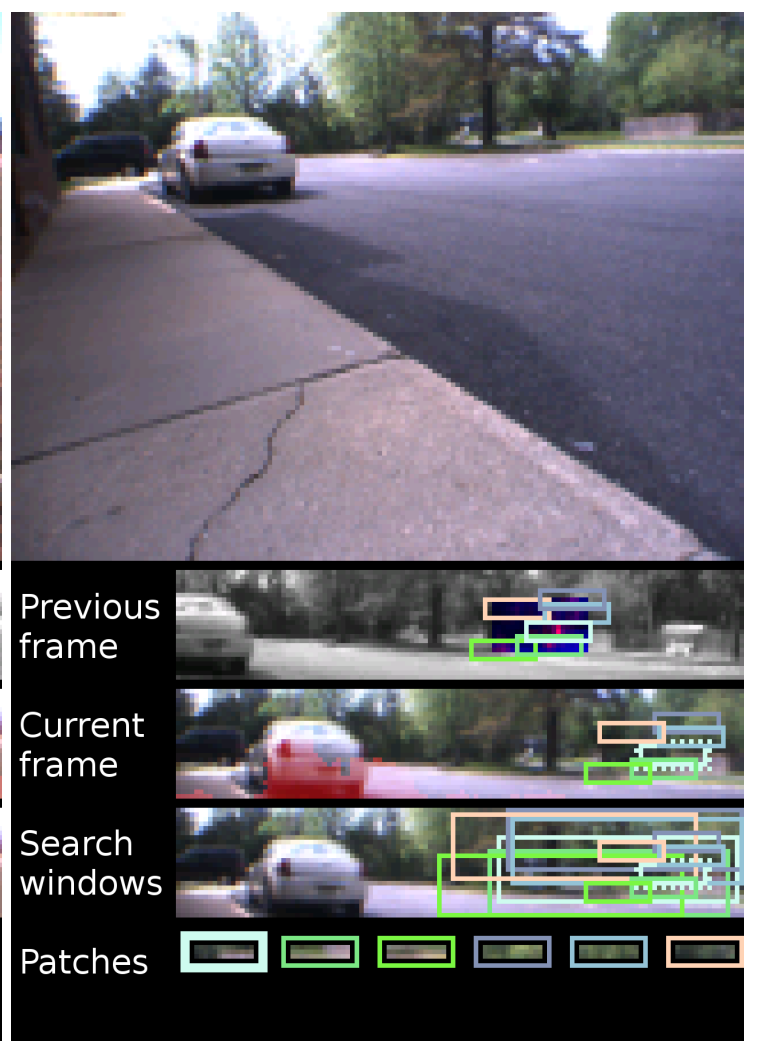

(b) Office parking lot

Figure 19: The robot's view, while running two of the courses in figure 24. In figure 19(a), at top is the current camera image, at the low resolution of 160 by 120 . Below this is the spherical horizon image of the previous frame. Harris corners are detected in a small window shown tinted in dark blue, with red pixels indicating Harris corner strength. This window is chosen either as the frontal direction (figure 19(a)) or the region that will be the frontal direction in the current frame, according to wheel odometry (figure 19(b)). Image patches (shown as small rectangles) are sampled around the strongest corners. The next image below shows the horizon of the current frame, with the image patches from the previous frame found using normalized cross-correlation (NCC). Each patch's NCC search is run in a window that spans the patch's position in the previous frame and the patch's predicted position in the current frame, as predicted by wheel odometry. These search windows are shown in the next image below, as rectangles surrounding each image patch. In figure 19(b) these search windows are wider because the robot is in the middle of a sharp turn. At the bottom are the isolated image patches. Figure 19(a) shows five good patches and one out-lier patch (dotted outline) that was rejected for disagreeing with the other patches about how much the robot rotated. Comparing the yellow rectangle in the previous frame and the current frame, we can see that it has shifted by one pixel relative to the other patches.

Most other work in relative pose tracking has focused either on using non-visual sensors to detect wheel slip, or on employing full visual odometry as a complete replacement to wheel odometry.

\subsubsection{Wheel odometry correction}

Wheel slip can occur in many flavors, from sudden spurts of wheel speed to gently increasing drift. The latter in particular is difficult to detect by simple cross-checking against motor current or inertial sensor output, requiring more nuanced approaches. (Ojeda et al., 2006) correct odometry using parameterized functions of motor current and soil cohesion. However, (Maimone et al., 2007) report that such an approach fares poorly unless the soil consistency is nearly homogeneous. Ward and Iagnemma train an SVM on hand-labeled odometric and inertial sensor outputs to detect immobilization (Ward and Iagnemma, 2007a). In another paper (Ward and Iagnemma, 2007b), the same authors employ a model-based approach, inferring robot velocity by fusing the output of a physical model with IMU, GPS, and wheel odometry output. A simpler approach to detect- 
ing slip would be to complement odometry with the absolute position measurements provided by GPS. Unfortunately, GPS input can be sporadic and inaccurate in wooded or urban environments (Sukkarieh et al., 1999) (Hofmann-Wellenhof et al., 2001), and even under optimal conditions refreshes only once per second. On our ground rover platform, we have found that one second is plenty of time for a robot to hallucinate a sharp turn due to wheel slip and react to it by sharply turning in the opposite direction. When driving alongside entangling vegetation or on narrow forest paths, such sudden "corrective" turns can prove catastrophic to a run. Furthermore, both GPS and odometry exhibit highly non-Gaussian error profiles, as neither GPS "jumps" nor wheel slip errors are well modeled as a random walk around a mean value. (Sukkarieh et al., 1999) therefore use a chi-squared gating function to detect and discard blatantly spurious sensor outputs, and feed only vetted sensor readings to a Gaussian model, in their case an EKF sensor fusion algorithm. However, this does not address the problem of GPS's low update frequency, nor a gating function's insensitivity to gradual odometry drift at low speeds. Rather than attempting to selectively detect and correct bad rotation estimates by the wheel odometer, we have opted to replace them entirely with the more reliable rotation estimates by our visual rotation tracker.

\subsubsection{Full visual odometry}

Full visual odometry tracks visual features to make a differential estimate of the robot's full 6-DOF pose. Problems that have been addressed in the past include include tracking and/or calibration from a small number of known landmarks (Triggs, 1999), performing triangulation with uncalibrated cameras (Stewenius et al., 2005a), and tracking or calibrating with minimal information (Stewenius et al., 2005b) (Schaffalitzky et al., 2000). Closer to our goals is the attempt to perform visual odometry in real time in natural scenery. (Nistér et al., 2004) tracked Harris corners (Harris and Stephens, 1988) in real time, discarding spurious feature associations between frames using RANSAC (Fischler and Bolles, 1981). Konolige et. al. improved upon this in their own realtime system by incorporating bundle adjustment to reduce drift (Agrawal and Konolige, 2007). However, their system occupies all of the processor time on a high-end CPU, requiring additional computers to handle other aspects of autonomous operation such as mapping and planning. The NASA Mars Exploration Vehicle is hit particularly hard by the computational demands of full VO, which can take up to 3 minutes per frame on its $20 \mathrm{MHz}$ processor, leading to an average movement of 10m/hour (Maimone et al., 2007). By contrast, our system is implemented on the same robotic platform used in (Agrawal and Konolige, 2007) where one of its two camera-computer pairs is dedicated to the task of real-time full VO. However, this leaves that camera-computer pair unable to perform other potentially critical tasks on its field of view, such as obstacle detection. For this reason, we have chosen our more lightweight approach.

Not all existing approaches to visual pose estimation attempt to calculate the full 6-DOF pose. Davison demonstrated that EKF-based SLAM algorithms, while slow when overloaded with landmarks, serve well in the limited domain of "visual compassing"(Montiel and Davison, 2006). In this scenario, the camera rotates in place, estimating its own orientation and the bearing and elevation of landmarks, without need of estimating their position. On our non-stationary platform, tracking landmarks for more than a few frames would have required estimating their position. Moreover, maintaining estimates of visual landmarks over long periods of time (Clemente et al., ) was of questionable utility in the LAGR contest, where prior locations were seldom revisited. To avoid implementing such a full 6DOF mapping algorithm, we chose to discard landmarks after a few frames, adopting an approach closer to visual odometry. 


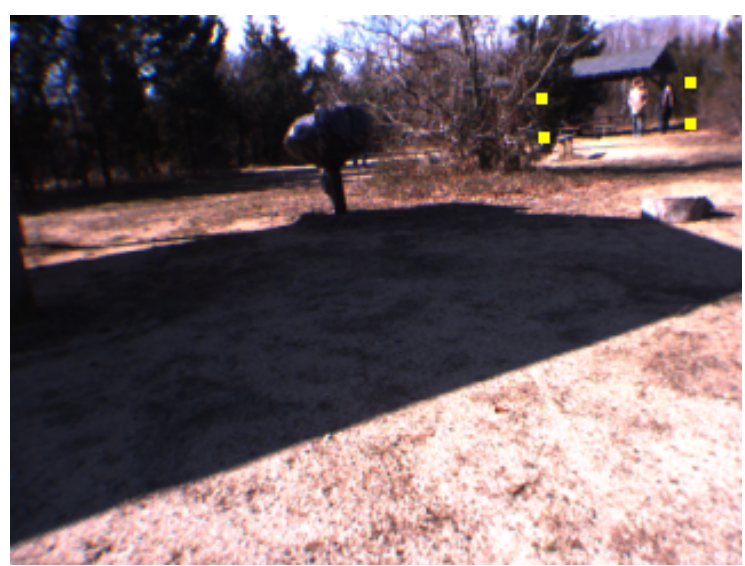

(a) Camera image

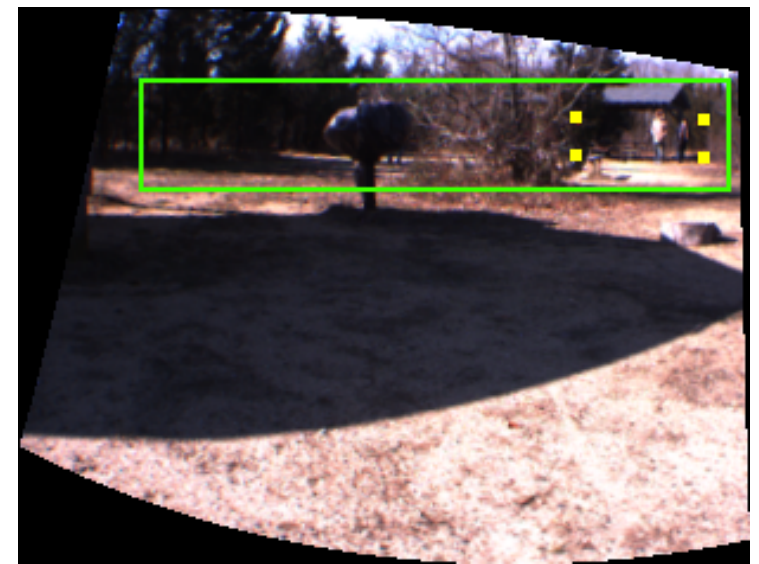

(b) Spherical image

Figure 20: A rectified camera image and its spherical transform. The asymmetry of the spherical image is due to the fact that the camera is pointed off to the left and down, with some axial roll. The robot is pointed straight at the area highlighted by four yellow dots, placed at pitch and yaw values $[0.03,0.1],[0.03,-0.1],[-0.03,-0.1]$, and $[-0.03,0.1]$, in radians. After the transformation, these points form an axis-aligned rectangle around the frontal direction. In practice, we transform only the portion of the camera image roughly located around the horizon, highlighted by the green rectangle above.

\subsubsection{Algorithm}

In the interest of keeping running costs down, our system tracks only six patches per frame, tracking a small number of wide-angle image patches at low resolution. The system samples patches from a region around the horizon, interpreting their horizontal motion from frame to frame as a rotation of the robot. There are two challenges to this approach: one is that a small number of features may be less robust to mismatches. The other is that a robot driving in a straight line will see features drift towards the sides of the image as it drives by ("drive-by drift"). Under a naïve implementation, such horizontal motion in the image would be incorrectly interpreted as a rotation. In this section we give a walk-through of our algorithm, paying particular attention to the solutions to the above problems. The overall algorithm is as follows:

1. Re-map the image to remove feature size distortions due to planar projection.

2. Sample features from a small region of the previous frame selected using wheel odometry.

3. Search for the sampled features in the current frame, again limiting the search area using wheel odometry.

4. Cross-validate the features' motions between frames and discard any outliers.

5. Localize the robot in the current frame by replacing the wheel odometry's rotation estimate with that of the visual odometer, and rotating the translation estimate by the difference in rotations.

\subsubsection{Re-map image to a spherical projection}

Because we track a small number of image patches per frame, care must be taken to minimize the number of mismatched patches. We use wide patches sub-tending eight degrees of yaw, as larger patches tend to be more distinctive. However, such large features stretch when moved from the center of the image to the edges, where each pixel subtends a smaller solid angle. This distortion can cause mismatches when searching for features that have moved from the center of the image 
to near an edge, or vice-versa. To remove this distortion, we re-map the camera image using a "spherical projection", where each pixel row and column subtends a fixed amount of vehiclerelative pitch and yaw $[\phi, \theta]$, respectively (figure 20 ). We define the mapping from camera image coordinates $[i, j]$ to spherical image coordinates $[k, l]$ as:

$$
\begin{aligned}
k(i, j) & =\left(\phi(i, j)-\phi_{o}\right) / a \\
l(i, j) & =\left(\theta(i, j)-\theta_{o}\right) / a
\end{aligned}
$$

Here, $a$ is a chosen ratio of radians per pixel, and $\left[\phi_{o}, \theta_{o}\right]$ is the chosen pitch and yaw of the view ray corresponding to the upper-left pixel in the spherical image. We choose $a$ as being the radiansper-pixel of the center pixel in the planar image. The center pixel subtends the largest angle of all the pixels, causing the spherically mapped image to be slightly smaller than the planar image. The spherical mapping is efficiently performed using a pre-calculated look-up table. To get the value of a pixel $[k, l]$ in the spherical image, one need only look up the the corresponding camera pixel indices in the table, and sample from that pixel in the camera image. We use the IPP (Stewart, 2004) function $\mathrm{Re}-\mathrm{map}$, which performs this mapping smoothly using bilinear interpolation. The $[i, j]$ corresponding to $[\phi, \theta]$ is calculated as:

$$
[i, j]=f\left(R_{c} R_{\theta} R_{\phi} \hat{\mathbf{z}}\right)
$$

Where $\hat{\mathbf{z}}$ is the unit vector in the forward direction in the vehicle's rotational coordinate frame, $R_{\phi}$ and $R_{\theta}$ are the rotation matrices corresponding to pitch and yaw, $R_{c}$ is the rotation from the vehicle frame to the camera frame, and $f$ is the projection function.

\subsection{Sampling features from previous frame}

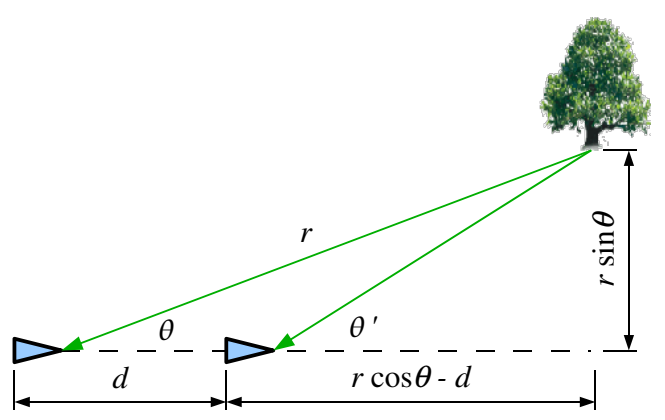

Figure 21: Parallax from pure forward motion. We wish to limit our attention to objects which will not shift under parallax from frame to frame. In a spherical image where each pixel subtends $\theta_{p}$ radians of yaw, this requires that the bearing $\theta$ of the object must not change more than $\theta_{p} / 2$. Given upper limits on $\theta$ and the frame-to-frame translation $d$, we may solve for a minimum distance $r$. We avoid parallax by detecting distance using stereo data calculated earlier for obstacle detection, and ignoring all features closer than $r$.

Even when the robot is moving straight forward, any feature except for those directly in front of the robot will drift towards the side of the image as the robot drives by. If we are to interpret the horizontal motion of features as robot rotation, we must limit our features to those whose "drive-by drift" between frames is less than half a pixel in the spherical image, and therefore undetectable. As shown in figure 21, this constraint defines a relation between the feature's distance $r$, bearing $\theta$, the maximum possible travel of the camera between frames $d$, and the yaw subtended by a pixel in the spherical image $\theta_{p}$ :

$$
r>\frac{d \tan \left(\theta_{p} / 2+\theta\right)}{\cos \theta \tan \left(\theta_{p} / 2+\theta\right)-\sin \theta}
$$

With a sufficiently reliable stereo vision system with which to estimate $r$, one could apply the above criteria to all features in the image, sampling image patches only around those features satisfying 
equation 17. However, stereo can be unreliable for large $r$, particularly at low image resolutions. Instead, we opt to limit our features to a small range of yaw $-\theta_{\max }<\theta<\theta_{\max }$ centered on the frontal direction $\theta=0$. We then plug $\theta_{\max }$ into equation 17 to derive a corresponding minimum distance $r_{\text {min }}$. Any feature closer than $r_{\text {min }}$ is deemed unfit for use. As discussed in section 5.4.2, when an insufficient number of viable landmarks are found in a particular frame, the pose for that frame is estimated using wheel odometry. In practice, this happens rarely outdoors. Even in dense forests such as the one in figure 24(c), hybrid VO "punted" on only $92.1 \%$ of the frames. we estimate distance using the dense stereo image already calculated by another component for the purposes of obstacle detection. If no such calculation is already being done, calculating stereo disparities over the entire image is an inefficient means of estimating feature distances. In this case, the approach of (Agrawal and Konolige, 2007) may be used, where stereo disparity is calculated for each patch rather than for each pixel.

The choice of $\theta_{\max }$ can be made based on the robot's expected speed, environment, and camera geometry. On our platform, the cameras point off to the sides, thereby making the frontal direction close to one side of the spherical image (see figure 20(b)). We therefore chose $\theta_{\max }$ to be the absolute value of the yaw of that side, namely \pm 0.09 or \pm 0.14 radians depending on the eye. This left $15 \%$ to $23 \%$ of the image available for sampling. Using these $\theta_{\max }$ values, along with a $d$ of 0.13 meters $(1.3 \mathrm{~m} / \mathrm{s} / 10 \mathrm{~Hz})$, equation 17 yields minimum distances $r_{\min }$ of $2.42 \mathrm{~m}$ and $3.69 \mathrm{~m}$. Note that $d$ is the maximum expected travel of the camera, which is not necessarily the maximum expected travel of the robot. On our robot the geometry and kinematics make the maximum vehicle travel a reasonable approximation to the maximum camera travel. Other platforms may require a different estimate of $d$.

The region defined by $-\theta_{\max }<\theta<\theta_{\max }$ is further shrunk on all sides by half the patch dimensions before applying a Harris corner detector to one of its channels. These corners are used to find suitable points to sample RGB patches from. The horizon images labeled "previous frame" in figure 19 show this region tinted in dark blue. As each image patch is selected, the Harris corners under that patch are set to zero as a form of non-max suppression. We sample 6 patches measuring $13 \times 3$ pixels, or 7.6 by 1.75 degrees of solid angle or $11 \%$ by $3 \%$ of the field of view.

\subsubsection{Searching for patches}

In outdoor environments, it is a common occurrence for the robot to move less than reported by wheel odometry ("wheel slip"). The opposite case of the robot moving significantly more than the wheels ("wheel skid") is far less common under vehicle speeds typically operated under by autonomous vehicles (Ward and Iagnemma, 2007b). We therefore trust our odometry to set an upper limit on the expected patch motion from frame to frame. When searching for image patches, we limit our search window to a region that encompasses the patch's position in the previous frame and the patch's position in the current frame, as predicted by wheel odometry. This window is inflated on all sides by half the patch dimensions for an added measure of safety. We apply a normalized cross-correlation of the image patch with this search window, and choose the maximum as the best-matching location.

\subsubsection{Cross-validate matches}

The change in yaw of an image patch from the previous frame to the current frame is that patch's estimate of the robot's rotation. To detect outliers, we cross-validate by having each patch "vote" for every other patch whose estimate differs by less than $\theta_{p}$. Patches whose vote tally is more than half the number of patches are deemed reliable, while others are rejected as outliers. The 
rotation estimate shared by all inliers is taken as the robot's rotation between the previous and current frames. When using a small number of patches, a pair of frames may occasionally present no patches with a sufficient number of votes. On such frames we let the wheel odometer supply the change in pose.

\subsubsection{Localizing the robot}

The robot's current pose is estimated as:

$$
p^{\prime}=p+R_{\frac{\theta_{v}}{2}} R_{-\theta_{w}} \Delta p_{w}
$$

where $p^{\prime}$ and $p$ are the current and previous pose estimates, $\Delta p_{w}$ is the change in pose as reported by wheel odometry, $\theta_{v}$ and $\theta_{w}$ are the change in yaw as reported by visual odometry and wheel odometry, and $R_{\text {angle }}$ is a rotation matrix representing a yaw rotation by angle. The concatenation $R_{\frac{\theta_{v}}{2}} R_{-\theta_{w}}$ expresses the fact that we undo the odometry-reported rotation $\theta_{w}$ and replace it with $\frac{\theta_{v}}{2}$.

We use the midpoint method to numerically integrate the pose forward, hence the use of $\frac{\theta_{v}}{2}$ rather than $\theta_{v}$.

\subsubsection{Implementation}

The visual odometer is implemented in Lush compiled to $\mathrm{C}$. We captured the camera images at a low resolution of $160 \times 120$ pixels, and used six image patches of $13 \times 3$ pixels. The Intel Performance Primitives (IPP) library (Stewart, 2004) was used for the spherical image transform, Harris corner detection, and normalized cross-correlation. The hybrid VO system runs within the same thread as the short-range stereo-based obstacle detector (Sermanet et al., 2007), running at $5-10 \mathrm{~Hz}$ as shown in figure 2 . The processor time is also shared by the long-range vision module running in a separate thread at $1 \mathrm{~Hz}$.

\section{Results}

\subsection{Architecture Results}

The mapping and planning algorithms used in this section are not the final ones described further, but the results described here are similar in the final system. Those intermediate algorithms were described in previous papers (Hadsell et al., 2007).

\subsubsection{Timing Results}

Fig. 22 plots the latencies and frequencies of an actual run of the robot for different layers of the system. In the top plot, the bottom curves indicate a camera and API latency of $190 \mathrm{~ms}$, i.e. the age of the data when it is available to our software on the eye machines. On top is the actuation latency of 250ms approximately, i.e. the age of the same data when it reaches the motors. The goal of Fast-OD or Layer 1 is to minimize this processing time between the sensors and the actuators in order to maximize reactivity. Similarly, an actuation latency of $700 \mathrm{~ms}$ was recorded for the Far-OD (Layer 2). Finally, Fast-OD exhibited a frequency of $10 \mathrm{~Hz}$ against $2-3 \mathrm{~Hz}$ for Far-OD. To conclude, those timing measures demonstrate Fast-OD's success to guarantee a low reactivity latency and a high frequency while letting Far-OD the possibility to run at a slower pace. The corresponding 

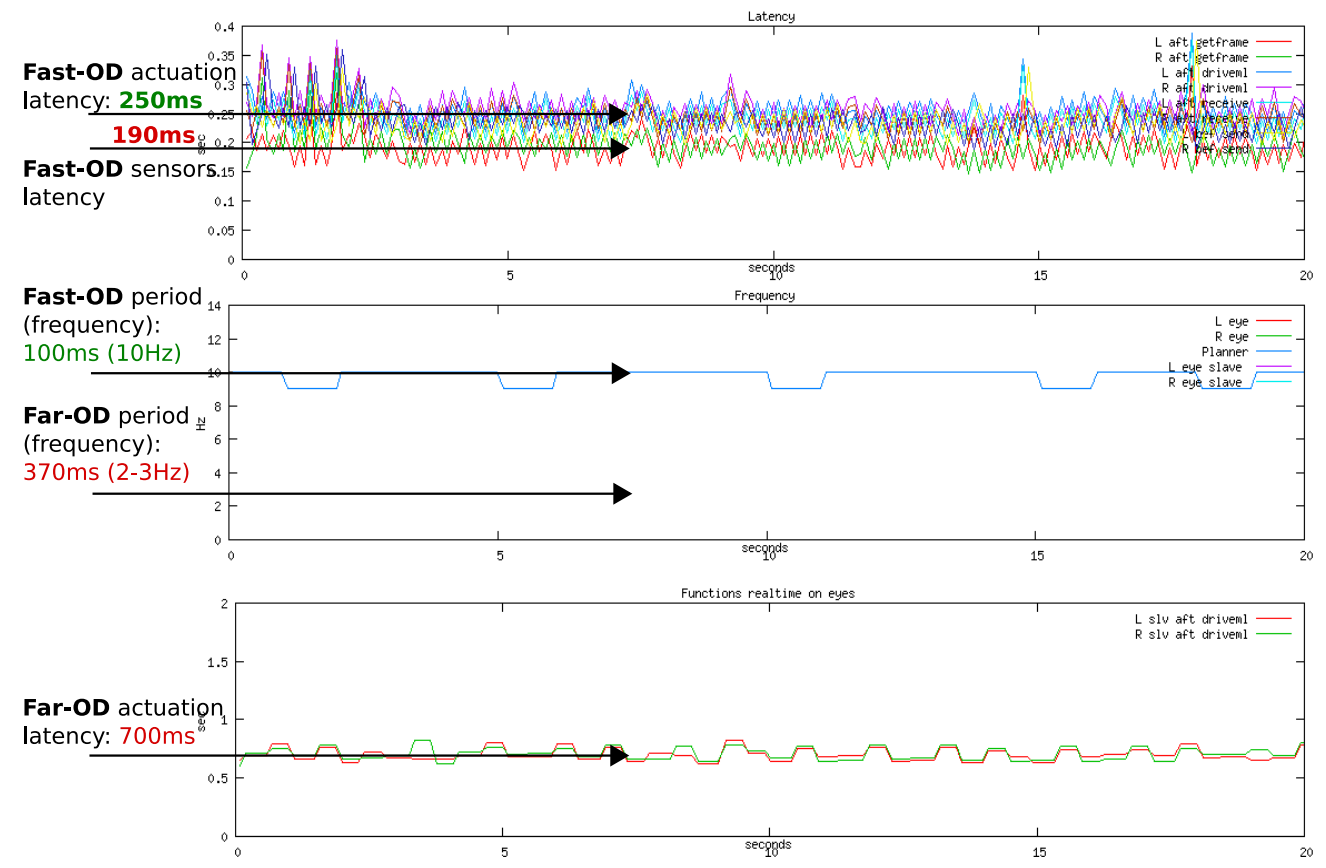

Figure 22: Internal timing plots. The top plot shows the age of the data at different processing stage. The data is already $190 \mathrm{~ms}$ old when received by the API, but only $250 \mathrm{~ms}$ old when delivered to the actuators by Fast-OD, whereas it is $700 \mathrm{~ms}$ old when delivered by Far-OD to the actuators (bottom plot). The middle plot exhibits the Fast-OD and Far-OD frequencies of $10 \mathrm{~Hz}$ and $2-3 \mathrm{~Hz}$ (The $2-3 \mathrm{~Hz}$ Far-OD frequency plot is not available in this particular figure but was observed in similar plots at the same period).

field performance is tested in the following experiment. Note again that those timing results were gathered during the middle of the LAGR program, as Layer 1 and 2 increased in complexity, frequencies dropped to $5-10 \mathrm{~Hz}$ for Layer 1 and $1 \mathrm{~Hz}$ for Layer 2 but remain effective.

\subsubsection{Field Results}

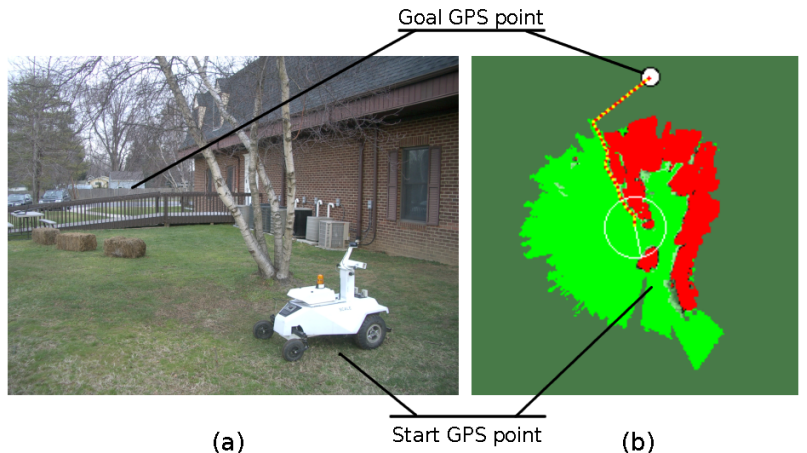

(a) (b)

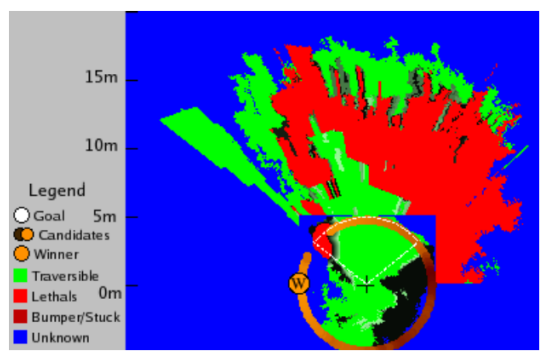

(c)

Figure 23: Test description. (a) Site of the field test. (b) Global map of the test produced by the robot. (c) Vehicle map produced by the robot at the entrance of the cul-de-sac. The vehicle map combines Fast-OD and Far-OD maps, thus we can see that thanks to the Far-OD, the winning candidate "W" of the Fast-OD map is chosen to avoid the cul-de-sac.

In order to show the benefits of our multi-layer architecture, the following test comparing different combination of Fast-OD and Far-OD was designed and is depicted in Fig. 23. Note however that a 320x240 stereo module was used as Far-OD in lieu of the neural network module because the latter was not mature enough at the time. First, to test reactivity, the robot starts a short distance from an obstacle and facing 90 degrees away from it. The goal is also 90 degrees away, so that 


\begin{tabular}{|c||c|c|c|c|c|c|}
\hline Test Mode & Runs & Frequency & Latency & $\begin{array}{c}\text { Total } \\
\text { Time }\end{array}$ & $\begin{array}{c}\text { Crashes } \\
\text { /Run }\end{array}$ & $\begin{array}{c}\text { Into Cul- } \\
\text { de-sac/Run }\end{array}$ \\
\hline \hline (1) Fast-OD only & 5 & Fast-OD: $10 \mathrm{~Hz}$ & $280 \mathrm{~ms}$ & $\mathbf{2 6 . 2 s}$ & 0 & 1 \\
\hline (2) Fast-OD+Far-OD in parallel & 5 & Fast-OD: 4-5Hz, Far-OD: $2 \mathrm{~Hz}$ & $300 \mathrm{~ms}$ & $\mathbf{1 9 s}$ & 0.2 & 0.2 \\
\hline (3) Fast-OD+Far-OD in series & 5 & Fast-OD+Far-OD: $3 \mathrm{~Hz}$ & $650 \mathrm{~ms}$ & $\mathbf{3 2 . 1 s}$ & 1.4 & 0 \\
\hline
\end{tabular}

Table 1: Average run results. The parallel system performed the fastest, with a nearly null number of collisions and entrances into the cul-de-sac.

the robot will turn right into the obstacle and avoid it or not depending on its reactivity. Second, to test long range vision, a cul-de-sac of hay-bales deeper than the Fast-OD radius is placed after the first obstacle and on the straight line to the goal. This way, a robot with no long-range or low frequency long-range vision will enter the cul-de-sac. In order to reach the goal the fastest in this test, a system has to combine fast reactivity and long-range vision.

Three different modes are tested: Fast-OD only, i.e. fast reactivity but no long-range vision, then Fast-OD and Far-OD in parallel, i.e. moderate reactivity and moderate long-range reactivity, and finally Fast-OD and Far-OD in series, i.e. poor reactivity but good long-range reactivity. Each configuration was ran 5 times and an average was taken for each metric and configuration.

Results are reported in Table 1. Configuration (1) had the highest frequency and lowest latency and never crashed but consistently ended-up into the cul-de-sac. (2) had low frequencies and good latency and crashed once in an obstacle. This crash probably happened because Fast-OD was running at a lower frequency compared to (1). It entered the cul-de-sac once, most likely because of Far-OD's lower frequency. This shows the limit of the low frequencies of Fast-OD and Far-OD and that a trade-off must be found. (2) reached the goal the fastest by having fewer crashes and going less into the cul-de-sac. Configuration (3) had a moderate frequency and low latency and many crashes were observed. However it consistently avoided the cul-de-sac. Note that latency is determined by sensors to actuators time and is not necessarily a factor of frequency, for example on the LAGR platform, the eye machines can run at $4 \mathrm{~Hz}$ but frame latency be more than $250 \mathrm{~ms}$ because it includes network time between eye and planner machines as well as planning time. Thus using Far-OD only (about $4 \mathrm{~Hz}$ ) would yield good frequency but poor latency, resulting in poor performance; however Fast-OD at $4 \mathrm{~Hz}$ does maintain good latency: latency and frequency have to be maintained together.

Table 1 indeed shows that Fast-OD alone performed very well in reactivity but not in long-range vision, and Fast-OD+Far-OD in series performed well in long-range but not in reactivity. The Fast-OD+Far-OD in parallel combination did not individually perform as well on each task but overall provided the best compromise between them.

Note that a reasonable balance between each module must be found to insure good results. One cannot, for example, let the Far-OD control loop latency and period be too high to keep good long-range reactivity. Depending on the speed of the vehicle, the processing time and maximum distance of each vision module, a trade-off must be found. One could also use more than two layers of perception and planning modules. For example, a mid-range vision module with a slow stereo system could be placed in between Far-OD and Fast-OD, thus allowing even more computation time for Far-OD.

\subsection{Trajectory Results}

We compare our system of learned vehicle dynamics to our previous system which uses a function of the steering angle to determine which wheel commands to apply. The vision, localization, mapping and planning of the two systems are identical only the low level driving commands (Layer 1) were changed. Our previous system performed adequately and was rated the first best performer in independent government tests at the end of Phase I of the LAGR project. But the lack of pre- 


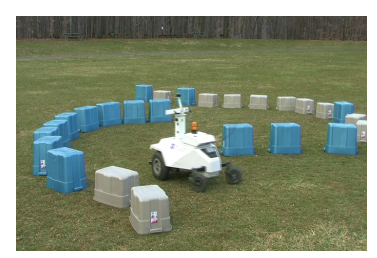

\begin{tabular}{|c|c|c|c|c|c|c|}
\hline \multirow[b]{2}{*}{ Course } & \multicolumn{2}{|c|}{ Running Time } & \multicolumn{2}{|c|}{ Obstacle hits } & \multicolumn{2}{|c|}{ Number of Runs } \\
\hline & steering & trajectories & steering & trajectories & steering & trajectories \\
\hline 1 wall (easy) & $19 \mathrm{~s}$ & $16 \mathrm{~s}$ & 0 & 0 & 1 & 1 \\
\hline 2 walls (easy) & $20 s$ & $15 \mathrm{~s}$ & 0 & 0 & 1 & 1 \\
\hline 3 walls (moderate) & $36.5 \mathrm{~s}$ & $18 \mathrm{~s}$ & 3 & 0 & 2 & 1 \\
\hline Slalom (hard) & $56 s$ & $26.33 \mathrm{~s}$ & 11 & 0 & 1 & 3 \\
\hline Scattered (hardest) & $\mathrm{X}$ & 24 & $\mathrm{X}$ & 0 & 0 & 1 \\
\hline
\end{tabular}

Table 2: Comparison of old steering-angle system (red) versus new learned-trajectories system (green). On the left is a picture of the "slalom" course. On the right, running times and number of collisions are reported. The new system with learned trajectories maintains a low running time over all courses and a total of 0 collisions. The trajectory bank used in this experiment was only $15 \%$ full and was extracted from 2 hours of human recorded runs.

cise vehicle dynamics caused collisions which are completely avoided with the new system. Both systems were run 1 to 3 times over 5 different 30 meter courses (Table 2), each consisting of a different arrangement of buckets as obstacles, with gradually increasing difficulty. Running times and number of bumper hits were recorded and averaged in Table 2. As the difficulty increases, using the old system (called "steering"), the running time and number of hits increases dramatically. The most difficult course could not be tested because the system would systematically destroy the bucket arrangement. The new system with learned trajectories, on the contrary, keeps a low running time over all courses and a total of 0 collisions. The driving capabilities of the system can be seen in video (Sermanet et al., 2008).

\subsection{Visual Odometry Results}

The hybrid VO system has been tested on various outdoor terrain including the area around an office building, an open field, and a narrow path through a forest. For the results presented here, we recorded logs in these settings, and ran both our hybrid VO and the full 6-DOF VO of (Agrawal and Konolige, 2007) on them, as a benchmark for accuracy and processor time. Fig. 24 shows the pose trajectories of three runs. Predictably, wheel odometry fused with IMU consistently fares the worst, accumulating small rotational errors that affect the entire trajectory that follows. The EKF fusion of wheel odometry, IMU, and GPS does better, except in the forest where the GPS signal can be both sporadic and inaccurate. Even with clear GPS reception as in fig. 24(b), the GPS-aided trajectory features a discontinuity at the top of the image due to satellites coming in and out of reception. In figure 24(b), the robot was driven around a sidewalk. At the end of the run, wheel spin was deliberately induced by attempting to drive up an intraversable curb. Naturally, all trajectories other than full VO end with a spurious short straight segment that represents the resulting hallucinated forward motion. In practice, we found such sharp discontinuities rare. Furthermore, devoting a $\mathrm{CPU}$ to full VO on our platform would have prevented one of the two stereo camera pairs from performing obstacle detection on its field of view. The plot in 24(c) shows the robot going down a narrow path through a forest. Accurate, high-frequency (i.e. inexpensive) estimates of the robot bearing are particularly important in such settings, where false rotations due to wheel slip are frequent, and can cause a robot to counter-steer into entangling obstacles on each side. The hybrid VO retraces the path accurately after a quick 180-degree turn.

The processor time per frame for each of these runs are reported in Table 3. The run-times shown are those of a Pentium 4M laptop at $2 \mathrm{GHz}$, running off of image and sensor data logged by the robot. The robot's CPUs are approximately 2.5 times faster. While our system does not track translations, it does use range information to rule out features that are too close to the robot. As discussed in section 5.4, we get this information "for free" by appropriating the stereo image already calculated by our obstacle classification system. If no such stereo image data is available, we can also adopt the approach of (Agrawal and Konolige, 2007), where a patch is searched for in the other stereo camera to estimate distance on a per-patch rather than per-pixel basis. In Table 3, 


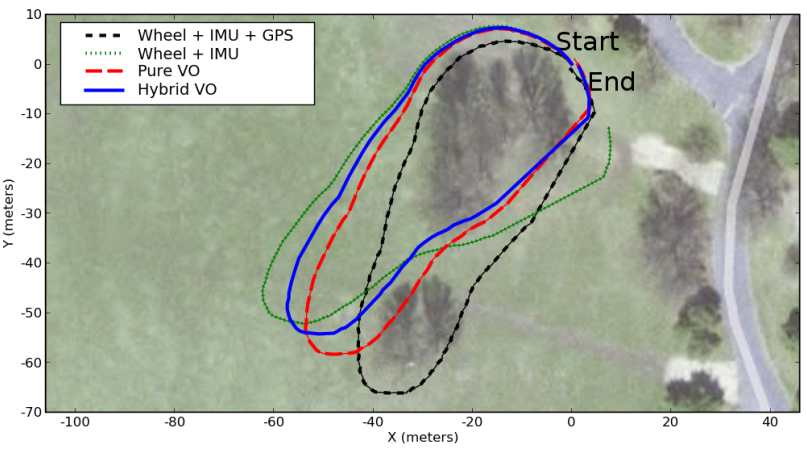

(a) Field Loop: A closed loop around two tree clusters.

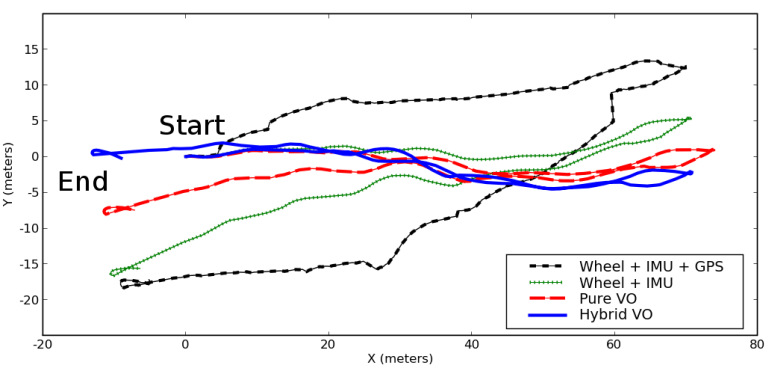

(c) Forest Path: Down a narrow forest path and back.

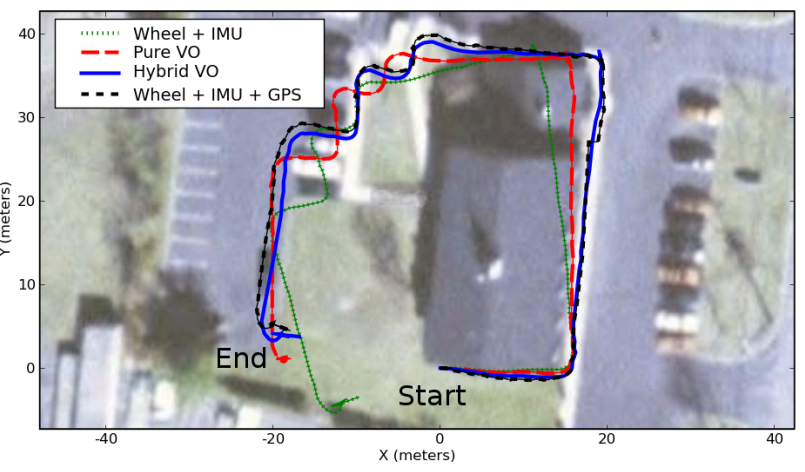

(b) Building Lap: A partial loop around a building.

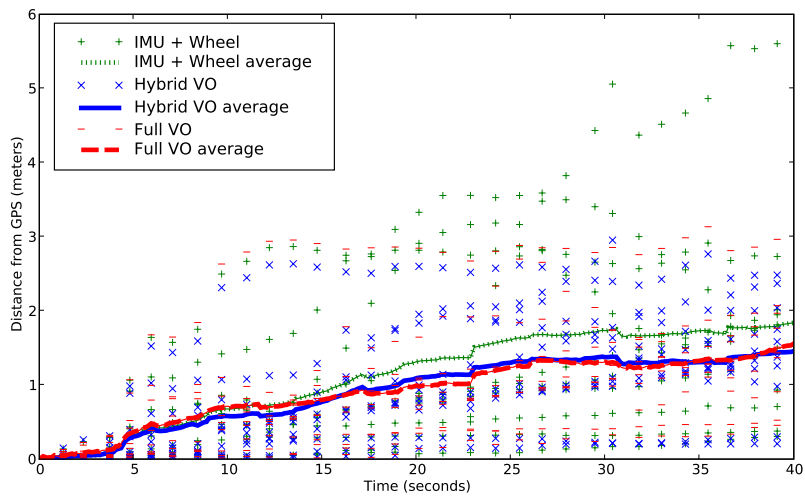

(d) Drift from GPS over time.

Figure 24: Vehicle trajectories, as measured using wheel odometry + IMU, GPS + wheel odometry + IMU, full VO (Agrawal and Konolige, 2007), and vision-corrected wheel odometry. In figure 24(a), the robot's initial pose and final pose are identical. The size of the opening in the loop can therefore be used as an indication of the correctness of the trajectories' shape. The non-GPS trajectories are globally oriented using the initial GPS orientation estimate, which can be noisy. In figure 24(b) the robot follows the sidewalk between the robot's first turn and sixth turn. The sidewalk's shape serves as ground truth during this segment. A typical GPS jump can be observed at the right side of the figure. In figure 24(c), the robot traverses a narrow forest path, then backtracks down the same path. GPS is inaccurate and disruptive in wooded areas such as these. Figure 24(d) Shows the drift from GPS over time. The dots show the distances of estimated trajectories from the GPS position for 10 randomly chosen runs. The lines show the corresponding averages over all runs. The average distance traveled (as measured by GPS) was 30.3 meters

the run-times for running just the hybrid VO, and for running the hybrid VO with per-patch stereo matching, are shown separately. The full VO runtime is best compared to the latter.

\subsection{Complete System Results}

We present experimental results obtained by running the robot on a outdoor courses with the longrange vision turned on and off and using the Baseline system. The goal is to show the benefits of each system against the other in end-to-end runs. The 3 navigation systems compared:

- Long-Range: our complete system with long-range vision enabled (100+m).

- Short-Range: same system as Long-Range but long-range vision is disabled, relying on the $5 \mathrm{~m}$ range reactive module.

- Baseline: the LAGR native system used as the 2004 state-of-the-art reference by the Government team, with approximately $15 \mathrm{~m}$ of vision range. 


\begin{tabular}{|c||c|c|c|}
\hline Log file & Full VO & Hybrid VO + range & Hybrid VO \\
\hline Field Loop & $266 \mathrm{~ms}$ & $11.5 \mathrm{~ms}$ & $8.0 \mathrm{~ms}$ \\
\hline Building Lap & $238 \mathrm{~ms}$ & $11.0 \mathrm{~ms}$ & $8.2 \mathrm{~ms}$ \\
\hline Forest Path & $153 \mathrm{~ms}$ & $14.0 \mathrm{~ms}$ & $9.3 \mathrm{~ms}$ \\
\hline
\end{tabular}

Table 3: CPU time per frame for full VO (Agrawal and Konolige, 2007) and hybrid VO on the three courses shown in figure 24. "Hybrid VO + range" shows the total time for calculating both stereo information and the pose estimate. "Hybrid VO" shows the time for the pose estimate only. Patch-based stereo, as performed by the full VO implementation, would result in a time between these two.

It is unfortunately not trivial to set up systematic field tests to gather statistically significant evidence because of the debugging-oriented character of most of the test runs during the development phase and limited time in a tight development and testing schedule. Also in general many little things make systematic testing harder such as limited robot and laptop battery power (recharging takes hours), hardware issues such as booting failures, logging disks failures, software logging failures, human factor during winter cold and summer heat. Therefore the complete runs results we present only count handfuls of runs but are however representative of our experience with development experiments toward the end of the development phase. Results are reported for 2 outdoor locations in New Jersey.

\subsubsection{Field Tests Location 1: Sandy Hook, New Jersey}
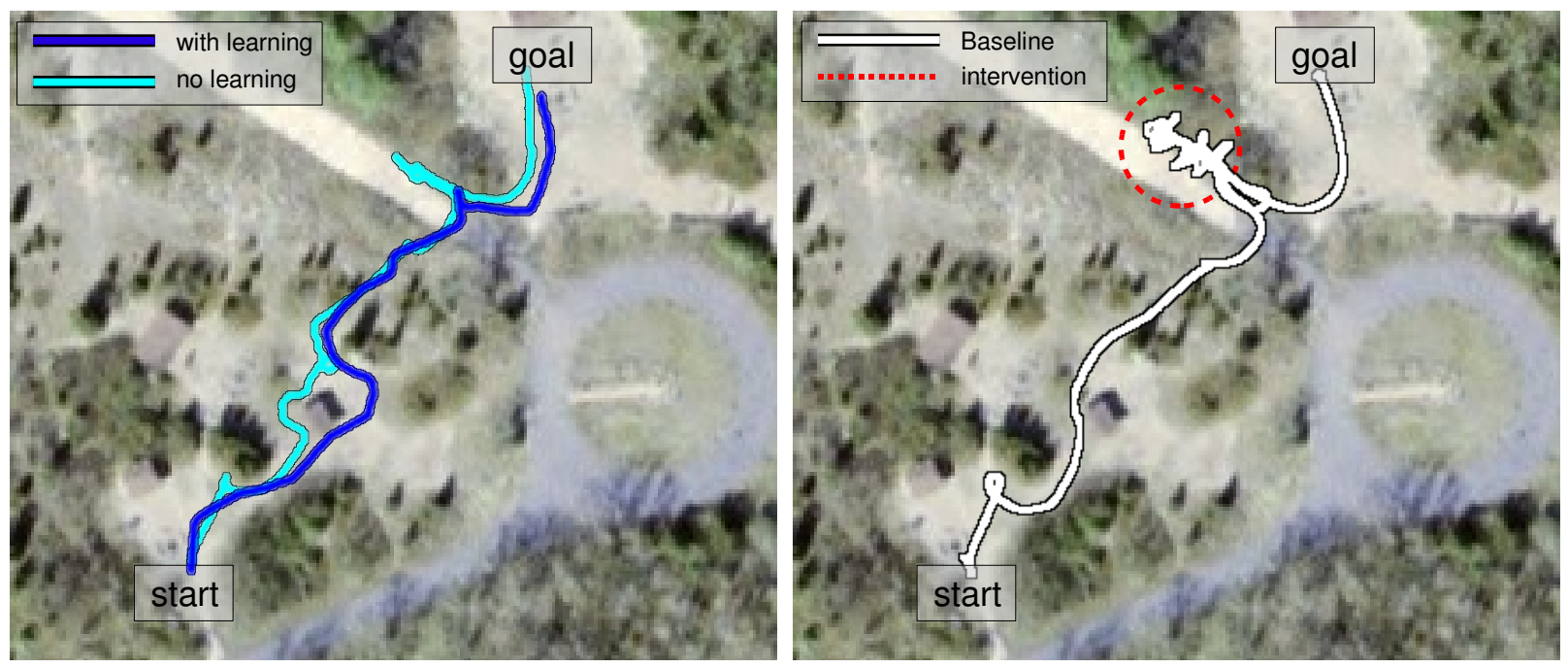

Figure 25: Aerial plots of comparison runs of long-range, short-range and Baseline systems. On the left are plotted the paths followed by our system with long-range vision enabled (dark blue) or disabled (Short-Range, light blue). The short-range vision explores a bit more to the left toward the end of the run as it lacks long-range vision. Those runs required no human intervention. On the right is the Baseline system which systematically got into trouble toward the end of the run and required several human interventions to finish the run.

\begin{tabular}{|c||c|c|c|c|}
\hline System & $\begin{array}{c}\text { Average } \\
\text { Time }\end{array}$ & $\begin{array}{c}\text { Average } \\
\text { Distance }\end{array}$ & $\begin{array}{c}\text { Human } \\
\text { Interventions }\end{array}$ & $\begin{array}{c}\text { Number } \\
\text { of Runs }\end{array}$ \\
\hline \hline Long-Range & $113.9 \mathrm{sec}$ & $122.2 \mathrm{~m}$ & 0 & 2 \\
\hline Short-Range Only & $123.7 \mathrm{sec}$ & $122.9 \mathrm{~m}$ & 0 & 1 \\
\hline Baseline & $364.6 \mathrm{sec}$ & $200.4 \mathrm{~m}$ & 2 & 2 \\
\hline
\end{tabular}

Table 4: Performance comparison of long-range, short-range and Baseline systems. The long-range system performed the fastest, closely followed by the same system with the long-range vision turned off, while the baseline took significantly more time to reach the goal and requiring 2 human interventions after getting stuck into bushes. 
The course (Fig. 25) consists of trees, bushes and few man-made obstacles. Results are reported in Table 4 with average distances to goal, average times to goal, number of human interventions and number of runs. The long-range vision performed the fastest and faster than the Baseline system by a factor of 3.2. The short-range system, even though much more short-sighted than the Baseline performed significantly better and almost as well as the long-range system. It is important to note that the traveled distance of the long-range system was almost identical in average to the shortrange system but the former still performed faster in average. In fact, the distances of the 2 longrange system runs were $113.79 \mathrm{~m}$ and $130.52 \mathrm{~m}$, averaging to $122.15 \mathrm{~m}$, and times were $108.70 \mathrm{~s}$ and $119.03 \mathrm{~s}$, averaging to 113.86 . This can be explained by the fact that even though the long-range system might have chosen longer paths than the short-range system, it made smooth decision and maintained a higher cruising speed, while the short-sighted robot making poor decisions had to stop or make stronger turns more often, thus increasing the total run time.

\subsubsection{Field Tests Location 2: Cross Farm Park, Holmdel, New Jersey}

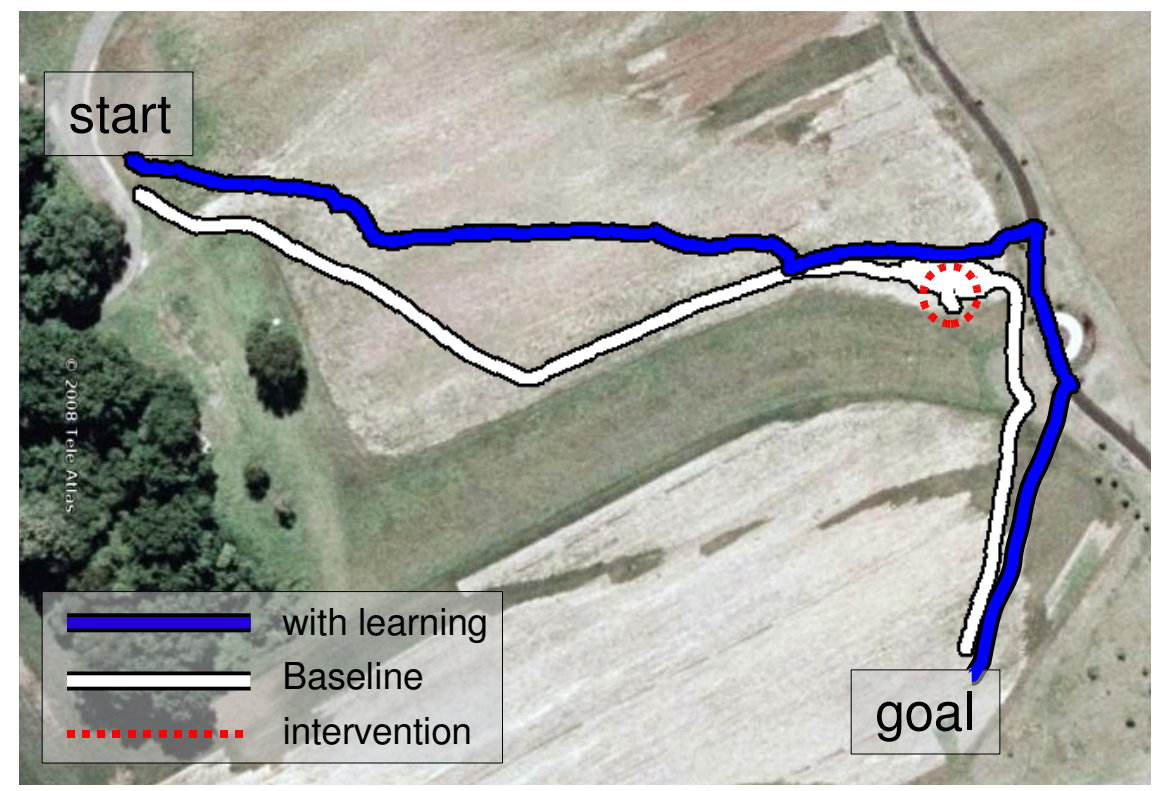

Figure 26: Aerial plots of comparison runs of long-range and Baseline systems. This aerial view is approximately 300 meters wide. The long-range system in blue clearly identifies the big tall-grass area from the beginning and stays out of trouble while the Baseline system got close to the grass and got into trouble.

\begin{tabular}{|c||c|c|c|c|}
\hline System & Time & Distance & $\begin{array}{c}\text { Human } \\
\text { Interventions }\end{array}$ & $\begin{array}{c}\text { Number } \\
\text { of Runs }\end{array}$ \\
\hline \hline Long-Range & $254.2 \mathrm{sec}$ & $314.0 \mathrm{~m}$ & 0 & 1 \\
\hline Baseline & $503.8 \mathrm{sec}$ & $479.2 \mathrm{~m}$ & 1 & 1 \\
\hline
\end{tabular}

Table 5: Performance comparison of long-range and Baseline systems. The long-range system performed almost twice faster than the Baseline and did not require any human intervention.

This run demonstrates the performance of the long-range system over the Baseline system, over a long distance (the width of the aerial view in Fig. 26 is approximately 300m). A wide tall-grass area separates the start and the goal points, the long-range system clearly identifies the far edge of this area from the beginning while the Baseline only steers away after reaching the grass. The Baseline also required human intervention after getting stuck in the grass. The long-range however stayed away from trouble and even identified the road as easily traversable, going out of its way to 
drive on it for a bit. From Table 5, the long-range system ran 198\% faster and travelled only $65 \%$ of the distance of the Baseline run.

\section{Future Work}

\subsection{Layer 3: Mapping and Planning}

The hyperbolic polar map provides an efficient solution to vision-based and vehicle-centered mapping. Nevertheless for courses longer than in LAGR tests, it needs to be coupled with an abstract global mapping as suggested by Layer 3 in Fig. 2. This Layer 3 has not been studied for this project, however a landmark recognition module for perception with a topological mapping and planning module seem to offer an elegant solution. Of course, the traditional Cartesian mapping remains easy to implement but its limitations (inefficient memory and computationally wise, prone to pose errors) should ultimately lead research to more abstract representations.

\subsection{Layer 2: Mapping}

The following current limitations of the h-polar mapping may be improved:

- Points coverage (merging and splitting): it is assumed that new data appears in front of the vehicle when moving forward, then merging with previous data. Points moving outside of the field-of-view into cells covering smaller areas scatter into tiny dots when they used to represent a bigger area. Merging and splitting heuristics may be improved to cope with those issues but it is not clear if it can solve it and for what price. However, this is in practice rarely an issue thanks to sub-sampling, smoothing and because points appear in front of the vehicle. Thus most points outside of the field-of-view, compress together when moving away from the center.

- Ground plane assumption: although 3D coordinates are kept internally, planning is executed in a flat $2 \mathrm{D}$ world. $\mathrm{Z}$ costs could be incorporated in the planning to reflect terrain relief.

- Hysteresis: an additional and fairly simple planning feature missing in the current system is planning stabilization through hysteresis. A lot of the instabilities sometimes observed during field testing are due to the inability to stick to a high-level planning decision, switching back and forth between different paths.

- Registration: h-polar provides an efficient space for registration of image patches. Registration techniques to cope with pose imprecisions should be investigated to improve the mapping precision.

\subsection{Layer 1: Trajectory Recording and Learning}

Besides exploring more difference measures to cover more of the trajectory search space as discussed above, another interesting way of research is to increase the autonomy of trajectory recording with online and off-line methods, in order to facilitate even more the recording process without any human intervention and to constantly optimize trajectories during navigation.

- Off-line Trajectories Search with Global Search Heuristics: Using a minimal set of basic recorded trajectories, an off-line algorithm could reconstruct new non-recorded tra- 
jectories from pieces of those basic trajectories. This way, all slots of the bank could get filled and optimized more easily. To speed up the search of optimized trajectories, global search heuristics such as genetic algorithms could be used to take advantage of the best trajectory pieces combinations.

- Online Trajectories Recording in Production Mode: When the vehicle runs in production mode, it is very cheap to record and add new trajectories on the fly when more optimized ones occur. With minimal effort, the robot can constantly improve its driving skills through experience.

- Online Trajectories Recording and Search in Self-supervised Mode: Given a large obstacle-free training area, the robot can try to fill its trajectory bank itself by knowing which areas need trajectories or optimization. During this self-supervised learning mode, the trajectory space could be searched using simple wheel commands heuristics and gradient descent to approach the desired target points.

\subsection{Layer 1: Visual Odometry}

We are currently working on using bearing-only VO to bootstrap an additional "translation-only VO" step that operates on very nearby patches at the bottom of the image. Such patches have usually been difficult to track, as small features on the ground are often indistinct, whereas larger areas of texture may require a rotation-invariant representation. Using the rotation estimate from bearing-only $\mathrm{VO}$, large ground patches may be rotated in the local ground plane to their expected orientations in the current frame. The distance to such nearby patches may be estimated with stereo vision at a reasonable accuracy even at low resolutions. We expect such a 3-DOF VO system to be far cheaper than existing VO methods, which estimate translation and rotation simultaneously, requiring higher resolutions.

\section{Conclusion}

A complete and robust software navigation system was developed providing collision-free and long-range navigation capabilities. The multi-layer perception, mapping and planning architecture handles the latency and frequency issues induced by sophisticated processing. It also conveniently separates the necessary different types of mapping and planning for different ranges, a Cartesian map with trajectory planning in the short-range and a hyperbolic polar map with grid-based planning in the long-range. The cheap but robust fast perception, planning and visual odometry layer provide the low-level stability required to support the sophisticated long-range vision. The maneuver dictionary and visual odometry contributed to the robust real-time navigation thanks to their simplicity and efficiency. Similarly to this paper, the video (Sermanet et al., 2008) explains and demonstrate this entire system.

Results show individual performance of key modules and overall performance as well. The complete system shows a systematic performance improvement over the reference Baseline system and over itself when long-range vision is turned off. Further research would mainly address coupling the current navigation layers with a global abstract layer for quasi-infinite global navigation.

\section{Acknowledgements}




\section{References}

Agrawal, M. and Konolige, K. (2007). Rough terrain visual odometry. Proceedings of the International Conference on Advanced Robotics (ICAR). 25, 28, 30, 34, 35, 36

Albus, J., Bostelman, R., Chang, T., Hong, T., Shackleford, W., and Shneier, M. (2006). Learning in a hierarchical control system: 4D/RCS in the DARPA LAGR program. In Journal of Field Robotics (JFR). 4

Andersen, E. and Taylor, C. (2007). Improving mav pose estimation using visual information. In Proc. of Int'l Conf on Intelligent Robots and Systems (IROS), pages 3745-3750. IEEE. 26

Angelova, A., Matthies, L., Helmick, D., and Perona, P. (2006). Slip prediction using visual information. In Proc. of Robotics: Science and Systems (RSS). 4

Angelova, A., Matthies, L., Helmick, D., and Perona, P. (2007). Fast terrain classification using variable-length representation for autonomous navigation. In Proc. of Conf. on Computer Vision and Pattern Recognition (CVPR). 4

Bajracharya, M., Tang, B., Howard, A., Turmon, M., and Matthies, L. (2008). Learning long-range terrain classification for autonomous navigation. In Proc. of Int'l Conf. on Robotics and Automation (ICRA). 4

Bayouth, M., Nourbakhsh, I., and Thorpe, C. (1998). A hybrid human-computer autonomous vehicle architecture. 7

Beetz, M. (2001). Structured reactive controllers. Autonomous Agents and Multi-Agent Systems, 4(1-2):25-55. 7

Behnke, S. (2004). Local multiresolution path planning. In Robocup 2003: Robot Soccer World Cup VII. 11

Bibby, C. and Reid, I. (2007). Simultaneous localisation and mapping in dynamic environments (slamide) with reversible data association. In Proc. of Robotics: Science and Systems (RSS). 25

Blas, M. R., Agrawal, M., Konolige, K., and Sundaresan, A. (2008). Fast color/texture segmentation for outdoor robots. In Proc. of Int'l Conf on Intelligent Robots and Systems (IROS). 4

Brooks, R. (1986). A robust layered control system for a mobile robot. In Robotics and Automation, IEEE Journal of [legacy, pre-1988]. 6

Brown, A. and Sullivan, D. (2002). Inertial navigation electro-optical aiding during gps dropouts. In Proceedings of the Joint Navigation Conference. 26

Bryson, A. E. and Ho, Y. C. (1975). Applied optimal control. In New York: Hemisphere Publishing. 20

Clemente, L., Davison, A., Reid, I., Neira, J., and Tards, J. Mapping large loops with a single hand-held camera. In Proc. of Robotics: Science and Systems (RSS). 28

Coombs, D., Murphy, K., Lacaze, A., and Legowik, S. (2000). Driving autonomously offroad up to $35 \mathrm{~km} / \mathrm{h}$. In Intelligent Vehicles Symposium. 21

Elfes, A. (1991). Occupancy grids: A stochastic spatial representation for active robot perception. In Autonomous Mobile Robots: Perception, Mapping, and Navigation (S. S. Iyengar and A. Elfes, eds.), IEEE Computer Society Press, pp. 6071.10

Fischler, M. A. and Bolles, R. C. (1981). Random sample consensus: a paradigm for model fitting with applications to image analysis and automated cartography. Commun. ACM, 24(6):381-395. 28

Frazzoli, E., Dahleh, M. A., and Feron, E. (2005). Maneuver-based motion planning for nonlinear systems with symmetries. In IEEE transactions on robotics. 21

Goldberg, S. B., Maimone, M., and Matthies, L. (2002). Stereo vision and robot navigation software for planetary exploration. In IEEE Aerospace Conf. Proc. 10, 19

Grudic, G., Mulligan, J., Otte, M., and Bates, A. (2007). Online learning of multiple perceptual models for navigation in unknown terrain. In 6th International Conference on Field and Service Robotics. 4

Hadsell, R., Sermanet, P., Ben, J., Erkan, A., Han, J., Flepp, B., Muller, U., and LeCun, Y. (2007). Online learning for offroad robots: Using spatial label propagation to learn long-range traversability. In Proc. of Robotics: Science and Systems (RSS). 11,32

Hadsell, R., Sermanet, P., Erkan, A., Scoffier, M., Kavukcuoglu, K., Muller, U., and LeCun, Y. (2008). Learning long-range vision for autonomous off-road driving. In Journal of Field Robotics (JFR). 2, 5, 10,19

Harris, C. and Stephens, M. (1988). A combined corner and edge detector,. In Proceedings of the 4th Alvey Vision Conference, pages 147-151. 28

Hofmann-Wellenhof, B., Lichtenegger, H., and Collins, J. (2001). Global Positioning System: Theory and Practice. SpringerVerlag, 5th edition. 26

Howard, T. and Kelly, A. (2005). Terrain-adaptive generation of optimal continuous trajectories for mobile robots. In International Symposium on Artificial Intelligence. 21

Kanayama, Y. and Hartman, B. (1988). Smooth local path planning for autonomous vehicles. In Technical Report, Dept. of Computer Science, University of California, Santa Barbara. 20

Kanayama, Y. and Miyake, N. (1985). Trajectory generation for mobile robots. In Robotics Research, MIT Press, Cambridge. 20

Kelly, A. and Nagy, B. (2003). Reactive nonholonomic trajectory generation via parametric optimal control. In International Journal of Robotics Research. 21

Kelly, A. and Stentz, A. (1998). Stereo vision enhancements for low-cost outdoor autonomous vehicles. ICRA Workshop WS-7. 10, 19 
Kelly, A., Stentz, A., Amidi, O., Bode, M., Bradley, D., Diaz-Calderon, A., Happold, M., Herman, H., Mandelbaum, R., Pilarski, T., Rander, P., Thayer, S., Vallidis, N., and Warner, R. (2006). Toward reliable off road autonomous vehicles operating in challenging environments. In The International Journal of Robotics Research, Vol. 25, No. 5-6, pp. 449-483. 4

Komoriya, K., Tachi, S., and Tanie, K. (1984). A method for autonomous locomotion of mobile robots. In Journal of the Robotics Society of Japan, vol 2, pp 222-231. 20

Kriegman, D. J., Triendl, E., and Binford, T. O. (1989). Stereo vision and navigation in buildings for mobile robots. IEEE Trans. Robotics and Automation, 5(6):792-803. 19

Longega, L., Panzieri, S., Pascucci, F., and Ulivi, G. (2003). Indoor robot navigation using log-polar local maps. In International IFAC Symposium on Robot Control. 11

Lookingbill, A., Lieb, D., and Thrun, S. (2007). Optical Flow Approaches for Self-supervised Learning in Autonomous Mobile Robot Navigation, pages 29-44. 4

Low, K. H., Leow, W. K., and Ang Jr., M. H. (2002). Integrated planning and control of mobile robot with self-organizing neural network. In Proc. IEEE International Conference on Robotics and Automation (ICRA'02), volume 4, pages 3870-3875. 7

Maimone, M., Cheng, Y., and Matthies, L. (2007). Two years of visual odometry on the mars exploration rovers: Field reports. Journal of Field Robotics, 24(3):169-186. 26, 28

Manduchi, R., Castano, A., Talukder, A., and Matthies, L. (2003). Obstacle detection and terrain classification for autonomous off-road navigation. Autonomous Robot, 18:81-102. 10

Montiel, J. M. M. and Davison, A. (2006). A visual compass based on slam. In IEEE Int'l. Conf. on Robotics and Automation (ICRA). 28

Nistér, D., Naroditsky, O., and Bergen, J. (2004). Visual odometry. Proc. of Conf. on Computer Vision and Pattern Recognition (CVPR), 01:652-659. 25, 28

Ojeda, L., Cruz, D., Reina, G., and Borenstein, J. (2006). Current-based slippage detection and odometry correction for mobile robots and planetary rovers. IEEE Transactions on Robotics and Automation, 22(2). 26

Ollis, M., Huang, W., and Happold, M. (2007). A bayesian approach to imitation learning for robot navigation. In Proc. of Int'l Conf on Intelligent Robots and Systems (IROS). 4

Oriolo, Ulivi, G., and Vendittelli, M. (1997). Fuzzy maps: A new tool for mobile robot perception and planning. In Journal of Robotic Systems, 14(3), 179-197. 10

PiedMonte, M. and Feron, E. (1999). Aggressive maneuvering of autonomous aerial vehicles: A human-centered approach. In International Symposium on Robotics Research. 20

Saxena, A., Schulte, J., and Ng, A. (2007). Depth estimation using monocular and stereo cues. In 20th International Joint Conference on Artificial Intelligence. 4

Schäfer, H., Hahnfeld, P., and Berns, K. (2007). Real-time visual self-localisation in dynamic environments. In Autonome Mobile Systeme. 26

Schaffalitzky, F., Zisserman, A., Hartley, R. I., and Torr, P. H. S. (2000). A six point solution for structure and motion. In Proceedings of the European Conference on Computer Vision, pages 632-648. 28

Sermanet, P., Ben, R. H. J., Naz, A., Beat, E., and Lecun, M. Y. (2007). Speed-range dilammas for vision-based navigation in unstructured terrain. In IFAC Symposium on Intelligent Autonomous Vehicles. IFAC. 32

Sermanet, P., Hadsell, R., Scoffier, M., Grimes, M., Ben, J., Erkan, A., Crudele, C., Muller, U., and LeCun, Y. (2008). DARPA LAGR program: Learning applied to long-range vision using a collision-free navigation platform. http://www.youtube.com/watch?v=lowcgokiRG8. In AAAI Video Competition. 34, 40

Sofman, B., Lin, E., Bagnell, J., Vandapel, N., and Stentz, A. (2006). Improving robot navigation through self-supervised online learning. In Proc. of Robotics: Science and Systems (RSS). 10

Spenko, M., Kuroda, Y., Dubowsky, S., and Iagnemma, K. (2006). Hazard avoidance for high-speed mobile robots in rough terrain. In Journal of Field Robotics. 21

Stavens, D. and Thrun, S. (2006). A self-supervised terrain roughness estimator for off-road autonomous driving. In Proc. of Conf. on Uncertainty in $A I(U A I) .3$

Stentz, A. (1994). Optimal and efficient path planning for partially-known environments. In Robotics and Automation (RA). 4

Stewart, E. (2004). Intel Integrated Performance Primitives: How to Optimize Software Applications Using Intel IPP. Intel Press. 29,31

Stewenius, H., Nister, D., Kahl, F., and Schaffalitzky, F. (2005a). A minimal solution for relative pose with unknown focal length. Computer Vision and Pattern Recognition, 2005. CVPR 2005. IEEE Computer Society Conference on, 2:789-794 vol. 2. 28

Stewenius, H., Schaffalitzky, F., and Nister, D. (2005b). How hard is 3-view triangulation really? pages I: 686-693. 28

Sukkarieh, S., Nebot, E., and Durrant-Whyte, H. (1999). A high integrity imu/gps navigation loop for autonomous land vehicle applications. In IEEE Transactions on Robotics and Automation, volume 15. 26, 27

Thrun, S. (2003). Exploring artificial intelligence in the new millennium, chapter Robotic mapping: a survey, pages 1-35. Morgan Kaufmann Publishers Inc., San Francisco, CA, USA. 25

Triggs, B. (1999). Camera pose and calibration from 4 or 5 known 3d points. In In Proc. 7th Int. Conf. on Computer Vision, pages 278-284. IEEE Computer Society Press. 28 
Tsumura, T., Fujiwara, N., Shirakawa, T., and Hashimoto, M. (1981). An experimental system for automatic guidance of a robotic vehicle following a route stored in memory. In Proceedings of the 11th International Symposium on Industrial Robots, pp 187-193. 20

Vernaza, P., Taskar, B., and Lee, D. D. (2008). Online, self-supervised terrain classification via discriminatively trained submodular markov random fields. In Proceedings of the IEEE International Conference on Robotics and Automation. 4

Veth, M. and Raquet, J. (2006). Two-dimensional stochastic projections for tight integration of optical and inertial sensors for navigation. In National Technical Meeting Proceedings of the Institute of Navigation, pages 587-596. 26

Veth, M., Raquet, J., and Pachter, M. (July 2006). Stochastic constraints for efficient image correspondence search. Aerospace and Electronic Systems, IEEE Transactions on, 42(3):973-982. 26

Wagner, M., Baird, J. C., and Barbaresi, W. (1980). The locus of environmental attention. J. of Environmental Psychology, 1:195206. 1,7

Ward, C. C. and Iagnemma, K. (2007a). Classification-based wheel slip detection and detector fusion for outdoor mobile robots. In Proc. of Int'l Conf. on Robotics and Automation (ICRA), pages 2730-2735. IEEE. 26

Ward, C. C. and Iagnemma, K. (2007b). Model-based wheel slip detection for outdoor mobile robots. In Proc. of Int'l Conf. on Robotics and Automation (ICRA), pages 2724-2729. IEEE. 26, 31

Zhang, H. and Ostrowski, J. (2002). Visual motion planning for mobile robots. In Robotics and Automation, IEEE Transactions. 11 